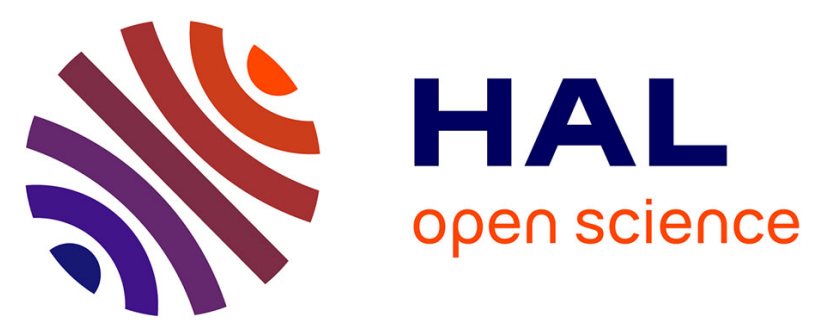

\title{
A homogenization approach for evaluating the longitudinal shear stiffness of reinforced soils: column versus cross trench configuration
}

\author{
Maxime Gueguin, Patrick de Buhan, Ghazi Hassen
}

\section{- To cite this version:}

Maxime Gueguin, Patrick de Buhan, Ghazi Hassen. A homogenization approach for evaluating the longitudinal shear stiffness of reinforced soils: column versus cross trench configuration. International Journal for Numerical and Analytical Methods in Geomechanics, 2013, pp.3150-3172. 10.1002/nag.2183 . hal-00907153

\section{HAL Id: hal-00907153 \\ https://hal-enpc.archives-ouvertes.fr/hal-00907153}

Submitted on 21 Nov 2013

HAL is a multi-disciplinary open access archive for the deposit and dissemination of scientific research documents, whether they are published or not. The documents may come from teaching and research institutions in France or abroad, or from public or private research centers.
L'archive ouverte pluridisciplinaire HAL, est destinée au dépôt et à la diffusion de documents scientifiques de niveau recherche, publiés ou non, émanant des établissements d'enseignement et de recherche français ou étrangers, des laboratoires publics ou privés. 


\title{
A homogenization approach for evaluating the longitudinal shear stiffness of reinforced soils: column $v s$. cross trench configuration
}

\author{
Maxime Gueguin, Patrick de Buhan ${ }^{(*)}$ and Ghazi Hassen
}

Université Paris Est, Laboratoire Navier (Ecole des Ponts ParisTech, IFSTTAR, CNRS

UMR 8205), 6 et 8 av. B. Pascal, 77455-Marne-la-Vallée, France

(*) Corresponding author: patrick.debuhan@enpc.fr

\begin{abstract}
.
The macroscopic linear elastic behaviour of inclusion-reinforced soils, regarded as periodic composite media, is investigated by means of the homogenization theory. Special attention is given here to the determination of their longitudinal shear stiffness properties, which strongly govern the reinforced ground response under lateral loading. Combining the use of analytical, variational as well as numerical methods, three particular engineeringrelevant configurations are more thoroughly examined: single trench, column and cross trench reinforcements. Fairly accurate closed-form expressions are thus obtained giving the value of the reinforced soil longitudinal shear stiffness as a function of the individual components shear moduli and reinforcement volume fraction. It is shown in particular that adopting a cross trench reinforcement layout instead of the classical column configuration results in a much higher improvement of the longitudinal shear stiffness. The results are then applied to assessing the reduction of soil liquefaction risk which can be attributed to the
\end{abstract}


presence of the reinforcing inclusions. Again, they clearly demonstrate the excellent performance of the cross trench configuration as compared to the complete inefficiency of the column reinforcement technique.

Keywords: reinforced soils; column reinforcement; trench reinforcement; shear stiffness; homogenisation; liquefaction risk. 


\section{Introduction}

As it is increasingly acknowledged today, soils reinforced by inclusions may be regarded as composite materials, the macroscopic behaviour of which can be determined using the same homogenization methods as those employed for instance, at a much smaller scale, for industrial fibre composite materials. The applicability of such methods is based on the fact that the characteristic length of the reinforcement, such as the spacing between two inclusions, is small in comparison with the overall dimensions of the geotechnical structure. Furthermore, far from being an arbitrary simplifying assumption, the condition of periodicity is perfectly representative of the actual soil reinforcement construction procedures.

As far as reinforcement by "thin" linear inclusions, made of much stiffer materials than the soil, like metal or concrete, is considered (metal strips or geotextiles in the earth reinforcement technique, soil nailing, rock bolting or even pile reinforced soils), a multiphase model is suitable for capturing the macroscopic behaviour of such reinforced soils (Sudret and de Buhan, 2001; Hassen and de Buhan, 2005). However, this model is not appropriate for describing the overall behaviour of foundation soils reinforced by large diameter columns (stone, lime or vibro-concrete inclusions), where a classical homogenization method for periodic media should therefore be preferred.

So far, the homogenization method has been mainly applied to this particular class of soils strengthened by columnar inclusions, in the context of the limit analysis or yield design approach, striving to determine a macroscopic anisotropic yield strength condition for the reinforced soil (Jellali et al., 2005, 2011). This method is also applied to derive a simplified lower bound approximation to the behaviour of stone column reinforced soils, in the elastic as well as plastic range (Balaam and Booker, 1981; Canetta and Nova, 1989; Lee and Pande, 
1998; Abdelkrim and de Buhan, 2007; Hassen et al., 2010). Focusing more specifically on the linear elastic behaviour, the latter approach gives reasonably good estimates for the axial stiffness characteristics of the reinforced soil, which are quite relevant for evaluating the settlements of the reinforced foundation under vertical loading. But they completely fail to correctly evaluate their stiffness characteristics under pure shear loading (longitudinal shear stiffness), which play a decisive role in the response of reinforced ground when subject for instance to earthquake induced lateral loading.

The purpose of the present contribution is therefore to obtain the most possible accurate estimates for such a longitudinal shear modulus of column reinforced soils, both constituents being considered as isotropic linear elastic materials with perfect bonding at their interface. Referring to reinforced soils as periodic elastic media, the analysis is entirely based upon the solution to a specific elastic boundary value problem, attached to the reinforced soil representative unit cell (section 2). Several configurations are then thoroughly examined in the light of such a method: multilayer or single trench reinforcement where exact closed form expressions are obtained for the longitudinal shear modulus (section 3); the most frequently used column configuration where analytical bounds are favourably compared to previous analytical (Hashin and Rosen, 1964) as well as numerical estimates (section 4); and finally the so called "cross trench" configuration which proves to be an optimal way of ground improvement, notably in terms of shear stiffness characteristics (section 5).

As a potential engineering application, section 6 describes how the previously obtained results can be incorporated into a simplified analysis of the way such reinforcements may contribute to reducing the liquefaction risk of saturated soils subject to earthquake loading. Among the main conclusions to be drawn from the analysis is the fact that no reduction of 
the liquefaction risk is resulting from reinforcing the soil by columnar inclusions, whereas a quite significant reduction could be expected from cross trench reinforcement.

\section{A brief outline of the elastic periodic homogenization method applied to reinforced soils}

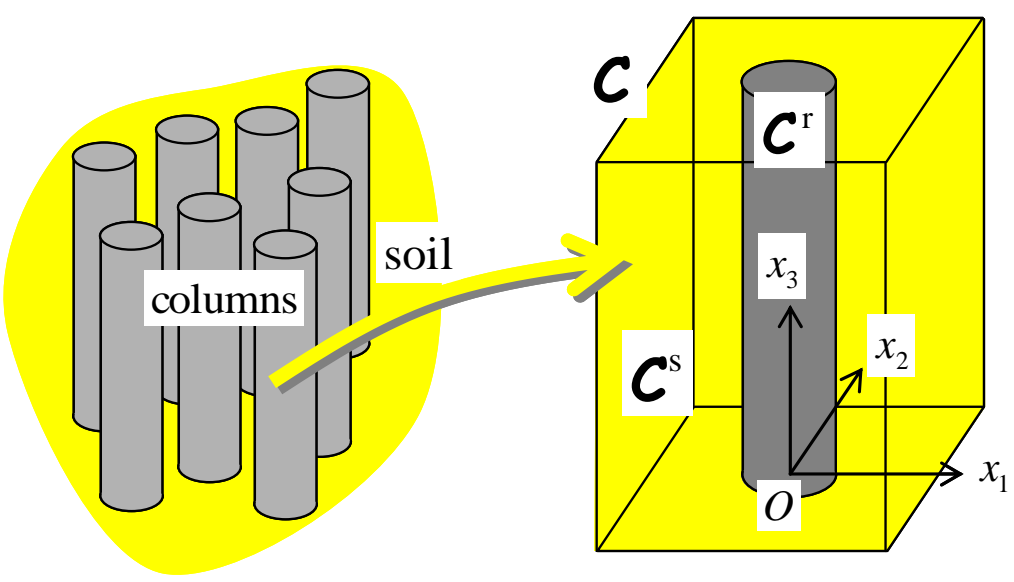

Figure.1. Soil reinforced by a group of columns and associated unit cell

A homogeneous soil reinforced by a group of vertical inclusions, parallel to the $O x_{3^{-}}$ direction, is considered. Owing to the fact that the inclusions are regularly placed into the soil, a representative cell, denoted by $C$, may be exhibited as shown in Figure 1 , and that both constituents are assumed to be elastic, the reinforced soil can thus be regarded as a periodic elastic medium. The homogenization theory applied to elastic periodic media has been developed for more than thirty years, notably by Sanchez-Palencia (1980), Duvaut (1976) or Bensoussan et al. (1978), based on the asymptotic expansion technique. It has been more recently extended to the elastoplastic behaviour of the materials (Suquet, 1985) with specific applications to column reinforced soils (Abdelkrim and de Buhan, 2007; Hassen et al., 2010). 
An important result has been established in the framework of this theory, according to which the determination of the macroscopic behaviour of the equivalent homogenous medium is obtained from the solution to an elastic boundary value problem, called the "auxiliary problem", defined on the representative cell of the periodic medium, considered as a micro-structure. This result states that the macroscopic elastic behaviour of the reinforced soil is defined as follows.

We first introduce the set $S$ of the statically admissible stress fields $\underline{\underline{\sigma}}$, on the one hand, the set $C$ of the kinematically admissible displacements fields $\xi$, on the other hand, defined as follows:

$$
\underline{\underline{\sigma}} \in S \Leftrightarrow\left\{\begin{array}{l}
\operatorname{div} \underline{\underline{\sigma}}(\underline{x})=0 \quad \forall \underline{x} \in C \\
\underline{\underline{\sigma}}\left(\underline{x}^{+}\right) \cdot \underline{n}\left(\underline{x}^{+}\right)=-\underline{\underline{\sigma}}\left(\underline{x}^{-}\right) \cdot \underline{n}\left(\underline{x}^{-}\right) \quad \forall \underline{x}^{ \pm} \in \partial C
\end{array}\right.
$$

and

$$
\underline{\xi} \in C \Leftrightarrow\left\{\begin{array}{lll}
\underline{\xi}(\underline{x})=\underline{F} \cdot \underline{x}+\underline{u}(\underline{x}) \quad \forall \underline{x} \in C \quad(a) \\
\underline{u}\left(\underline{x}^{+}\right)=\underline{u}\left(\underline{x}^{-}\right) & \forall \underline{x}^{ \pm} \in \partial C & (b)
\end{array}\right.
$$

Condition (1a) expresses the classical equilibrium equation in the absence of body force density, which is to be completed by the condition that the stress vector should remain continuous across possible stress discontinuity surfaces like the soil/column interface, while condition $(1 b)$, called antiperiodicity condition, states that stress vectors applied to the cell's external boundary $\partial C$ at two points $\underline{x}^{+}$and $\underline{x}^{-}$, deduced from one another by periodicity, are opposite. Likewise, definition (2) means that any kinematically admissible displacement field is the sum of a homogenous strain field, characterized by its gradient $\underline{\underline{F}}$, and a periodic fluctuation $\underline{u}$. 
On the basis of the above definitions, the general solution of the auxiliary problem can be formulated in the following way. Given any macroscopic stress $\underline{\underline{\Sigma}}$, which could be regarded as a "loading parameter" on the unit cell, one should find a statically admissible stress field and a kinematically admissible displacement field such that:

$$
\begin{gathered}
\underline{\underline{\Sigma}}=\langle\underline{\underline{\sigma}}\rangle, \underline{\underline{\sigma}} \in S, \underline{\xi} \in C, \\
\underline{\underline{\sigma}}(\underline{x})=\mathbf{C}(\underline{x}): \underline{\underline{\varepsilon}}(\underline{x}) \quad \text { with } \underline{\underline{\varepsilon}}(\underline{x})=1 / 2\left\{\underline{\underline{\operatorname{grad}}}(\underline{\xi})+{ }^{T} \underline{\underline{\operatorname{grad}}}(\underline{\xi})\right\}(\underline{x}), \forall \underline{x} \in C
\end{gathered}
$$

where $\langle *\rangle$ denotes the average value of $*$ over the unit cell $C$ and $\mathbf{c}(\underline{x})$ denotes the fourth order tensor of elastic moduli at point $\underline{x}$ of the unit cell. The macroscopic elastic law simply writes in the form of the following linear stress-strain relationship:

$$
\underline{\underline{\Sigma}}=\mathbf{c}^{\text {hom }}: \underline{\underline{\epsilon}} \text { with } \underline{\underline{\epsilon}}=\langle\underline{\underline{\varepsilon}}\rangle
$$

where $\mathbf{c}^{\text {hom }}$ represents the macroscopic elastic stiffness tensor.

An equivalent procedure consists in solving the same problem where the macroscopic strain, instead of the macroscopic stress, is prescribed a loading parameter to the unit cell.

\section{Longitudinal shear modulus of reinforced soil: the multilayer model}

Our analysis will from now on be focused on the evaluation of one particular component of $\mathbf{c}^{\text {hom }}$, namely the longitudinal shear stiffness $G_{\boldsymbol{L}}$ of the reinforced soil, defined as:

$$
\Sigma_{13}=\Sigma_{31}=\mathbf{c}_{1313}^{\text {hom }} \in_{13}=\mathbf{c}_{3131}^{\text {hom }} \in_{31}=2 G_{L} \in_{13}
$$

and reducing to the classical shear modulus (Lamé constant) for an isotropic elastic material. This modulus can be explicitly calculated in the case of a homogeneous soil (shear modulus 
$G_{s}$ ) reinforced by regularly spaced vertical "trenches" (layers) of a reinforcing material (shear modulus $G_{\boldsymbol{r}}>G_{\boldsymbol{s}}$ ) as sketched in Figure 2. Two configurations should be considered.

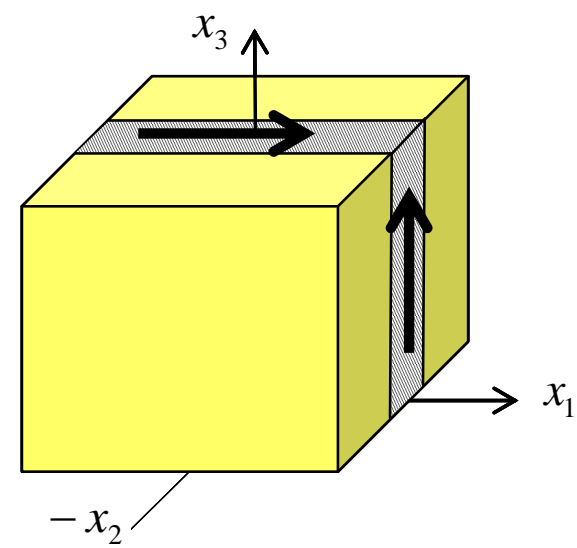

(a)

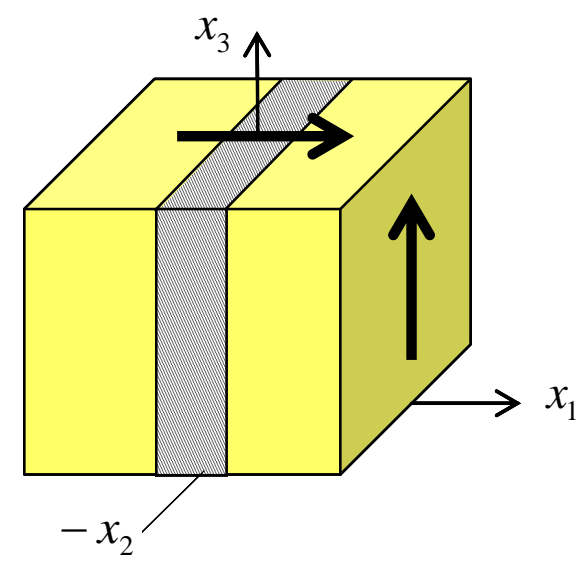

(b)

Figure 2. Homogeneous soil reinforced by parallel trenches subject to longitudinal shear

a) The reinforcing layers are parallel to the plane $O x_{1} x_{3}$ (Figure 2(a)). Implementing the previously described procedure, a macroscopic shear strain of the form:

$$
\underline{\underline{\epsilon}}=\Gamma\left(\underline{e}_{1} \otimes \underline{e}_{3}+\underline{e}_{3} \otimes \underline{e}_{1}\right)
$$

is imposed to the unit cell. It can be easily seen that the following displacement field (bottom of figure 3(a)):

$$
\underline{\xi}=2 \Gamma x_{1} \underline{e}_{3}
$$

is kinematically admissible with the macroscopic strain, while the following piecewise constant stress field (top of figure 3(a)):

$$
\underline{\underline{\sigma}}=\left\{\begin{array}{l}
2 G_{r} \Gamma\left(\underline{e}_{1} \otimes \underline{e}_{3}+\underline{e}_{3} \otimes \underline{e}_{1}\right) \text { in the reinforcing layer } \\
2 G_{s} \Gamma\left(\underline{e}_{1} \otimes \underline{e}_{3}+\underline{e}_{3} \otimes \underline{e}_{1}\right) \quad \text { in the soil }
\end{array}\right.
$$


is associated with the homogeneous strain field derived from (7) through the respective elastic constitutive laws of the soil and the reinforcement, while being statically admissible with the following macroscopic stress:

$$
\underline{\underline{\Sigma}}=\langle\underline{\underline{\sigma}}\rangle=2\left[(1-\eta) G_{s}+\eta G_{r}\right] \Gamma\left(\underline{e}_{1} \otimes \underline{e}_{3}+\underline{e}_{3} \otimes \underline{e}_{1}\right)
$$

hence from (5):

$$
G_{L}^{=}=(1-\eta) G_{s}+\eta G_{r}=\langle G\rangle
$$

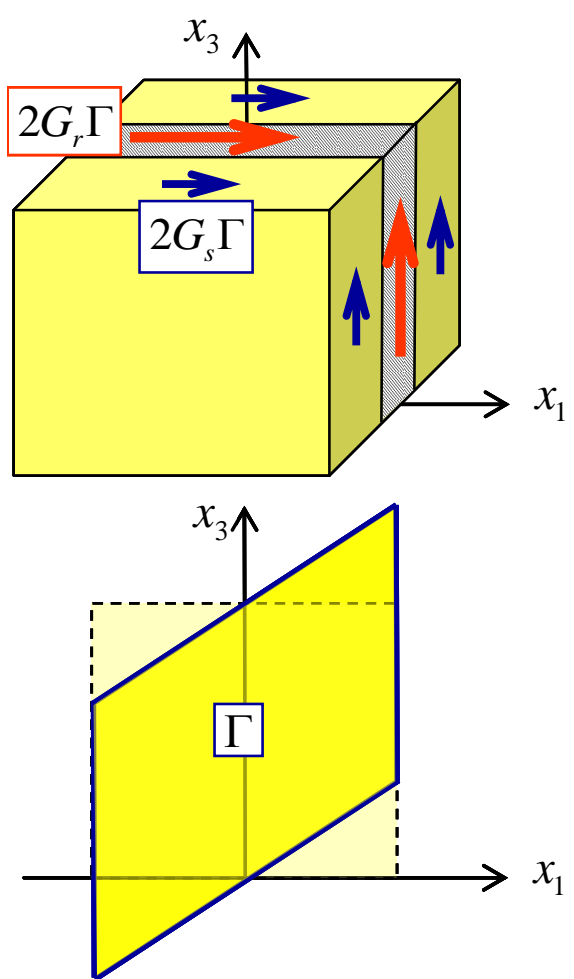

(a)

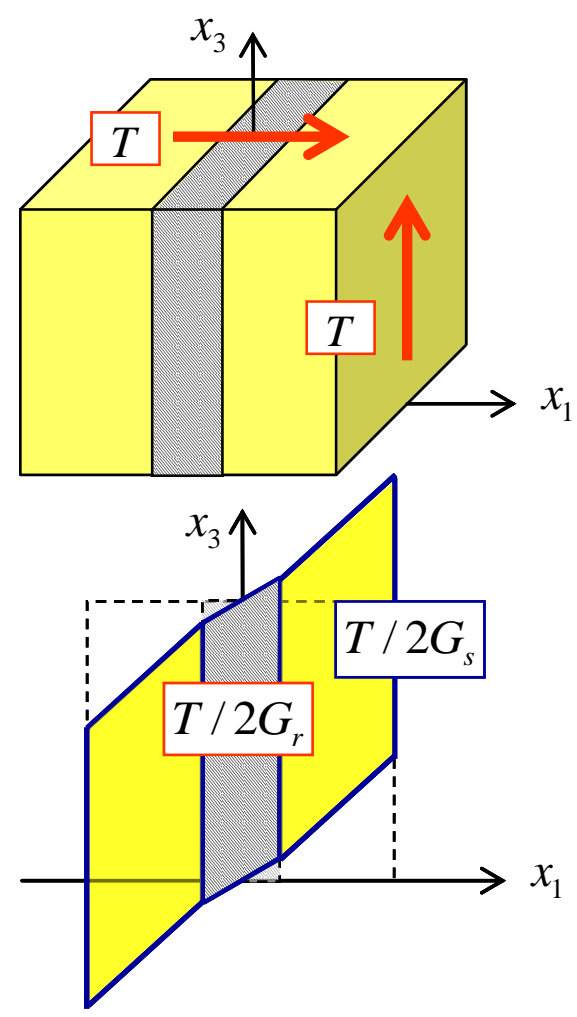

(b)

Figure 3. Stress fields and corresponding deformed configurations of a multilayered soil subject to longitudinal shear loading

where $\eta$ denotes the reinforcement volume fraction. It is to be noted that this exact result coincides with the classical Voigt upper bound for a two-phase composite. 
$b$ ) The reinforcing layers are perpendicular to the $O x_{1} x_{3}$ loading plane (Figure $2(b)$ ). A macroscopic shear stress of the form (top of figure 3(b)):

$$
\underline{\underline{\Sigma}}=T\left(\underline{e}_{1} \otimes \underline{e}_{3}+\underline{e}_{3} \otimes \underline{e}_{1}\right)
$$

being imposed to the unit cell, the homogeneous stress field $\underline{\underline{\sigma}}=\underline{\underline{\Sigma}}$ is obviously statically admissible and the piecewise constant strain field derived from the respective constituents elastic laws:

$$
\underline{\underline{\varepsilon}}=\left\{\begin{array}{l}
\left(T / 2 G_{r}\right)\left(\underline{e}_{1} \otimes \underline{e}_{3}+\underline{e}_{3} \otimes \underline{e}_{1}\right) \text { in the reinforcing layer } \\
\left(T / 2 G_{s}\right)\left(\underline{e}_{1} \otimes \underline{e}_{3}+\underline{e}_{3} \otimes \underline{e}_{1}\right) \text { in the soil }
\end{array}\right.
$$

may be easily integrated into the following piecewise linear displacement field (bottom of figure 3(b)):

$$
\underline{\xi}= \begin{cases}-\left[\left(T / 2 G_{r}\right) \eta / 2+\left(T / 2 G_{s}\right)\left(x_{1}+\eta / 2\right)\right] \underline{e}_{3} & \text { for }-1 \leq x_{1} \leq-\eta / 2 \text { (soil) } \\ \left(T / 2 G_{r}\right) x_{1} \underline{e}_{3} & \text { for }\left|x_{1}\right| \leq \eta / 2 \text { (reinforcement) } \\ {\left[\left(T / 2 G_{r}\right) \eta / 2+\left(T / 2 G_{s}\right)\left(x_{1}-\eta / 2\right)\right] \underline{e}_{3}} & \text { for } \eta / 2 \leq x_{1} \leq 1 \quad \text { (soil) }\end{cases}
$$

where the side of the cubic unit cell has been conventionally taken equal to unity. Since the corresponding macroscopic strain is:

$$
\underline{\underline{\epsilon}}=\langle\underline{\underline{\varepsilon}}\rangle=\left[(1-\eta)\left(T / 2 G_{s}\right)+\eta\left(T / 2 G_{r}\right)\right]\left(\underline{e}_{1} \otimes \underline{e}_{3}+\underline{e}_{3} \otimes \underline{e}_{1}\right)
$$

it follows from (5) that:

$$
1 / G_{L}^{\perp}=(1-\eta) / G_{s}+\eta / G_{r} \text { or } G_{L}^{\perp}=\langle 1 / G\rangle^{-1}
$$

which is identical with the classical Reuss lower bound. 


\section{The case of column-reinforced soil}

The most commonly encountered case of a soil reinforced by a group of circular cylindrical columnar inclusions is now investigated, trying to evaluate the longitudinal shear modulus in the same way as it has been done in the previous section. Since exact solutions are not available in such a configuration, the problem will be dealt with by resorting to analytical bounds derived from variational principles as well as to finite element simulations.

\subsection{Derivation of an upper bound for $G_{L}$}

It can be easily proved that, given any macroscopic strain $\subseteq$, and any displacement field $\underline{\xi}$, kinematically admissible with $\underline{\underline{ }}$, the following inequality, deduced from the minimum principle of the potential energy, holds:

$$
\begin{aligned}
& \forall \underline{\underline{\underline{\epsilon}}}, \forall \underline{\xi} \text { k.a. with } \underline{\underline{\epsilon}} \\
& 1 / 2 \underline{\underline{E}}: \mathbf{c}^{\mathrm{hom}}: \underline{\underline{\epsilon}} \leq\left\langle 1 / 2 \underline{\underline{\varepsilon^{\prime}}}(\underline{x}): \mathbf{C}(\underline{x}): \underline{\underline{\varepsilon}}^{\prime}(\underline{x})\right\rangle
\end{aligned}
$$

It follows that for a macroscopic shear strain of the form (6), we obtain an upper bound value for the macroscopic longitudinal shear modulus. Indeed:

$$
1 / 2 \subseteq: \mathbf{C}^{\text {hom }}: \subseteq=2 G_{L} \Gamma^{2}
$$

so that:

$$
\begin{gathered}
\forall \underline{\xi} \text { 'k.a. with } \underline{\underline{\epsilon}}=\Gamma\left(\underline{e}_{1} \otimes \underline{e}_{3}+\underline{e}_{3} \otimes \underline{e}_{1}\right) \\
G_{L} \leq\left\langle\underline{\varepsilon}^{\prime}(\underline{x}): \mathbf{C}(\underline{x}): \underline{\varepsilon}^{\prime}(\underline{x})\right\rangle / 4 \Gamma^{2}
\end{gathered}
$$

This inequality is implemented making use of the following displacement field:

$$
\underline{\xi}^{\prime}=\varphi(r) \cos \theta \underline{e}_{3}
$$


with (Figure 4 where, due to symmetries, only a quarter of the unit cell cross section of side equal to one has been represented):

$$
\varphi(r)=\mid \begin{aligned}
& \frac{2 G_{s}}{\left(G_{r}+G_{s}\right)-4 \rho^{2}\left(G_{r}-G_{s}\right)} r \quad \text { in zone }(I) \\
& \frac{\left(G_{r}+G_{s}\right) r-\frac{\rho^{2}}{r}\left(G_{r}-G_{s}\right)}{\left(G_{r}+G_{s}\right)-4 \rho^{2}\left(G_{r}-G_{s}\right)} \\
& r \quad \text { in zone }(I I I)
\end{aligned}
$$

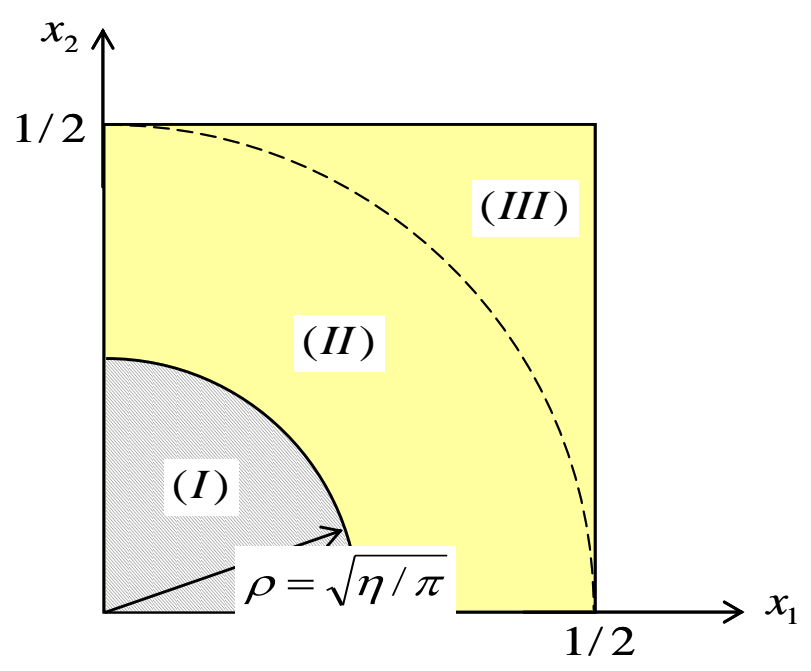

Figure 4. Zoning of the unit cell for the definition of kinematically (resp. statically) admissible displacement (resp. stress) fields

where $\rho$ is the column radius equal to $\sqrt{\eta / \pi}$ and $\left(r, \theta, x_{3}=z\right)$ the cylindrical coordinates. As a result of computations which may be found in Appendix A, the final result writes:

$$
G_{L} \leq G_{L, \mathrm{col}}^{\mathrm{ub}}=G_{s}\left[1+\frac{2 \eta\left(G_{r}-G_{s}\right)}{\left(G_{r}+G_{s}\right)-(4 \eta / \pi)\left(G_{r}-G_{s}\right)}\right]
$$




\subsection{Derivation of a lower bound estimate for $G_{L}$}

The derivation of such a lower bound is based on the minimum principle of the complementary energy applied to the same auxiliary problem, where a macroscopic stress $\underline{\underline{\Sigma}}$ is prescribed as loading parameter. For any stress field $\underline{\underline{\sigma^{\prime}}}$ statically admissible with $\underline{\underline{\Sigma}}$, this principle may be written as follows:

$$
\begin{gathered}
\forall \underline{\underline{\Sigma}}, \forall \underline{\underline{\sigma}}^{\prime} \text { s.a. with } \underline{\underline{\Sigma}} \\
1 / 2 \underline{\underline{\Sigma}}: \mathbf{s}^{\mathrm{hom}}: \underline{\underline{\Sigma}} \leq\left\langle 1 / 2 \underline{\underline{\sigma}}^{\prime}(\underline{x}): \mathbf{s}(\underline{x}): \underline{\underline{\sigma}}^{\prime}(\underline{x})\right\rangle
\end{gathered}
$$

where $\mathbf{s}^{\text {hom }}=\left(\mathbf{C}^{\text {hom }}\right)^{-1}$ and $\mathbf{s}(\underline{x})=(\mathbf{C}(\underline{x}))^{-1}$ are the macroscopic and local elastic compliance tensors, respectively. For a pure shear stress of the form (11), this inequality becomes:

$$
\begin{gathered}
\forall \underline{\underline{\sigma}}^{\prime} \text { s.a. with } \underline{\underline{\Sigma}}=T\left(\underline{e}_{1} \otimes \underline{e}_{3}+\underline{e}_{3} \otimes \underline{e}_{1}\right) \\
1 / 2 \underline{\underline{\Sigma}}: \mathbf{s}^{\text {hom }}: \underline{\underline{\Sigma}}=\mathbf{s}_{1313}^{\text {hom }} T^{2}=\frac{T^{2}}{2 G_{L}} \leq\left\langle 1 / 2 \underline{\underline{\sigma}}^{\prime}(\underline{x}): \mathbf{s}(\underline{x}): \underline{\underline{\sigma}}^{\prime}(\underline{x})\right\rangle
\end{gathered}
$$

which yields the following lower bound estimate for $G_{L}$ :

$$
\begin{gathered}
\forall \underline{\underline{\sigma}}^{\prime} \text { s.a. with } \underline{\underline{\underline{\Sigma}}}=T\left(\underline{e}_{1} \otimes \underline{e}_{3}+\underline{e}_{3} \otimes \underline{e}_{1}\right) \\
G_{L} \geq T^{2}\left\langle\underline{\underline{\sigma}}^{\prime}(\underline{x}): \mathbf{s}(\underline{x}): \underline{\underline{\sigma}}^{\prime}(\underline{x})\right\rangle^{-1}
\end{gathered}
$$

An appropriately selected stress field, described in Appendix A, allows deriving the following lower bound for the longitudinal shear modulus of a column reinforced soil:

$$
G_{L} \leq G_{L, \mathrm{col}}^{\mathrm{lb}}=G_{s}\left[1-\frac{2 \eta\left(G_{r}-G_{s}\right)}{\left(G_{r}+G_{s}\right)+(4 \eta / \pi)\left(G_{r}-G_{s}\right)}\right]^{-1}
$$




\subsection{Comparison with numerical and Hashin-Rosen estimates.}

Analytical upper and lower bounds (21) and (25) are now compared with numerical simulations carried out with the standard finite element code Cast3M (Cast3M, 2003) on the one hand, the Hashin-Rosen's estimate (Hashin and Rosen, 1964; Hashin, 1983), on the other hand.

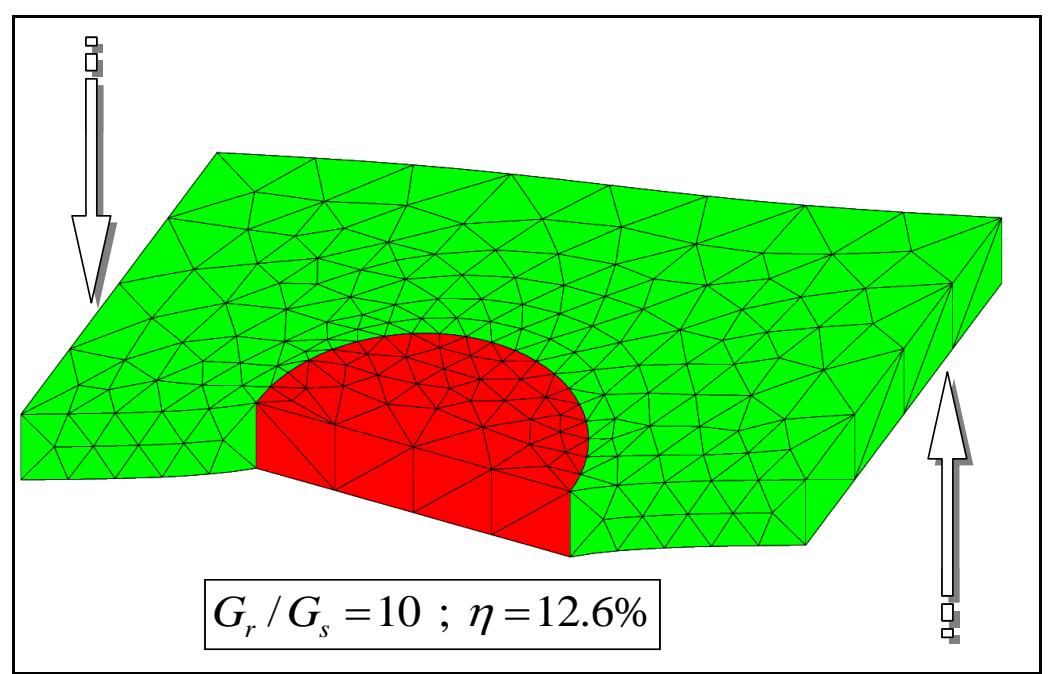

Figure 5. Elastically deformed configuration of a column reinforced soil under longitudinal shear loading

Since the reinforced soil is periodic in the horizontal cross sectional $O x_{1} x_{2}$-plane, implying that the solution to the auxiliary problem is independent of the $x_{3}$ coordinate, only a "slice" of the unit cell needs to be considered in the numerical analysis. Figure 5 pictures the deformed configuration of a column reinforced soil (exaggerated for the sake of clarity) under longitudinal shear loading, for typical values of the parameters, namely a shear stiffness of the column material $\left(G_{r}\right)$ ten times greater than that of the soil $\left(G_{s}\right)$ and a reinforcement volume fraction equal to $\eta=12.6 \%$. Due to the obvious symmetry property of the problem with respect to the loading plane $O x_{1} x_{3}$, half of the structure is represented in this figure. As it can be clearly seen from this figure, the column 
undergoes much smaller deformations that the surrounding soil in nearly the same way as for a single trench reinforcement when the reinforcing layer is perpendicular to the plane of loading (Figure 3(b)).

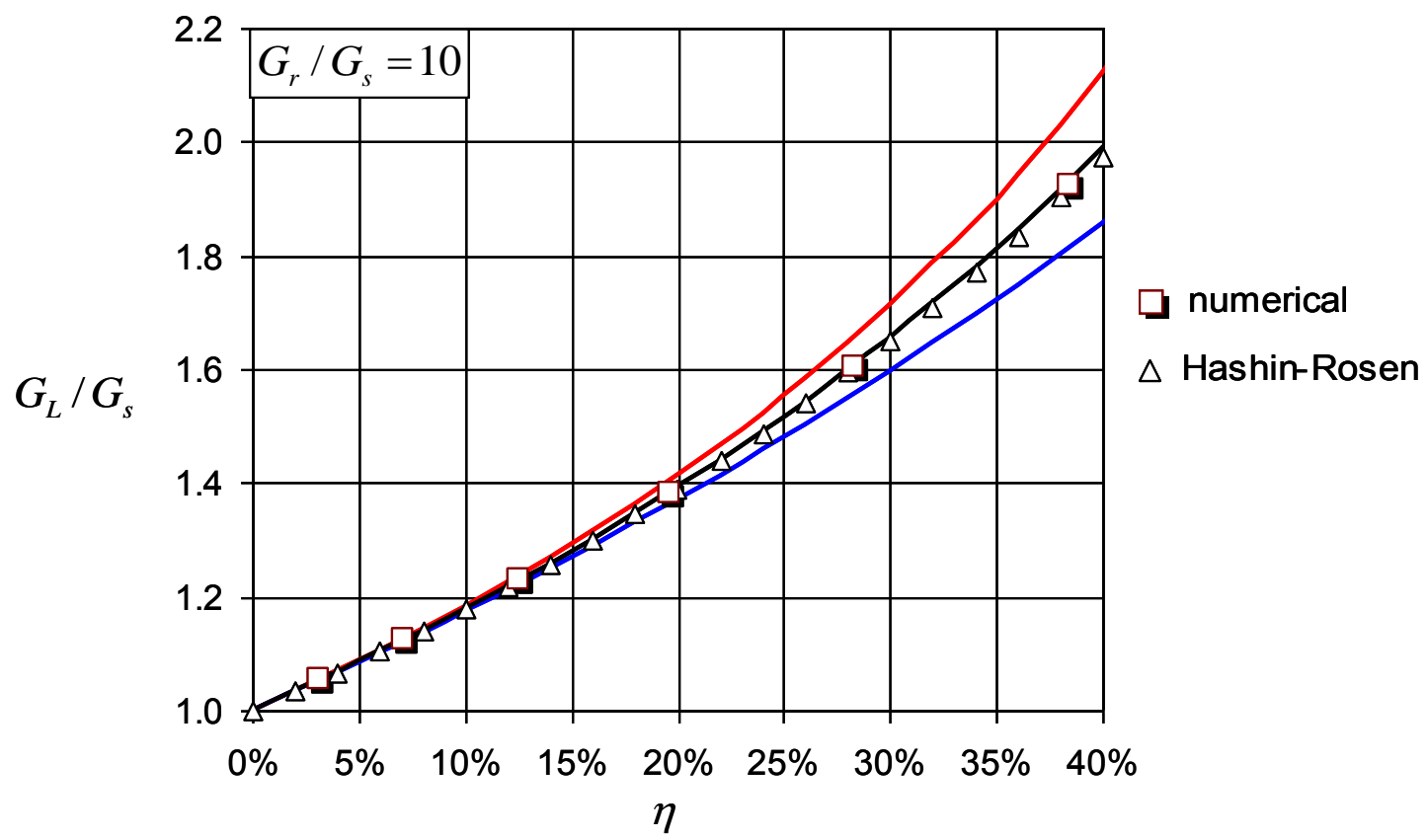

Figure 6. Comparison among different estimates for the longitudinal shear modulus of a column reinforced soil

The different analytical as well as numerical evaluations of the longitudinal shear modulus should also be compared with the analytical estimate provided by Hashin and Rosen (1964) or Hashin (1983) in a slightly different context. Indeed, according to their heuristic approach, the selected representative volume was not the cubic unit cell of periodic homogenization, but a composite circular cylinder made of a circular fiber (column) of radius $a$ surrounded by an annular cylinder of matrix (soil) of external radius equal to $b$, so that the reinforcement volume fraction is $\eta=(a / b)^{2}$. The effective longitudinal shear modulus they derived from such a morphologic model, is based on the elastic solution to such a composite cylinder problem, which may be found in Appendix 
A (the composite cylinder corresponds to the sub domain made of zones $(I)$ and $(I I)$ ). The corresponding estimate of the composite longitudinal shear modulus is:

$$
G_{L}^{\mathrm{HR}}=G_{s} \frac{\left(G_{r}+G_{s}\right)+\eta\left(G_{r}-G_{s}\right)}{\left(G_{r}+G_{s}\right)-\eta\left(G_{r}-G_{s}\right)}
$$

The different analytical bounds or estimates are compared to the results of finite element simulations in Figure 6 , in the case when $G_{r}=10 G_{s}$, the reinforcement volume fraction $\eta$ being varied between 0 and $40 \%$ (the engineering practice shows that this parameter rarely exceeds $30 \%$ ). According to this figure, the Hashin-Rosen estimates (triangular points) are very close to the numerical estimates (square bullets), both being comprised between the previously determined bounds (upper and lower solid curves) and almost coincident with the average value of upper and lower bounds (middle curve). It can be concluded that either the Hashin-Rosen estimate (26) or the half sum of bounds (21) and (25) provide a very accurate assessment for the increase of the longitudinal shear stiffness to be expected from reinforcing a soil by cylindrical columns.

\section{The cross trench configuration}

As shown in Figure 7, the increase in shear stiffness due to reinforcement by columnar inclusions is relatively limited, far closer to the Reuss lower bound (15) than to the Voigt upper bound (10). By way of example, for a reinforcement volume fraction $\eta$ equal to $20 \%$, the longitudinal shear stiffness is increased by about $40 \%$, which is twice as much as for a single trench configuration where the reinforcement is normal to the loading plane (bottom curve), but considerably less than for a single trench reinforcement placed in the loading plane (top curve), where such an increase would amount to $180 \%$. Indeed in the latter configuration, the shear stiffness of the reinforcing material is fully mobilized 
providing a kind of maximum "bracing effect" to the overall shear response of the reinforced soil.

This suggests that a potentially innovative and optimal reinforcement technique (which is actually beginning to develop in practice), would consist in improving the soil not by a single array of parallel trenches, but by a network of two perpendicular arrays of trenches, forming a kind of "honeycomb structure" embedded in the soil, as sketched in Figure 8: this particular reinforcement geometry will be called the cross trench configuration. Denoting by $t$ the thickness of a trench, and assuming that the side of the unit cell is taken equal to unity, the reinforcement volume fraction is simply:

$$
\eta=t(2-t)
$$

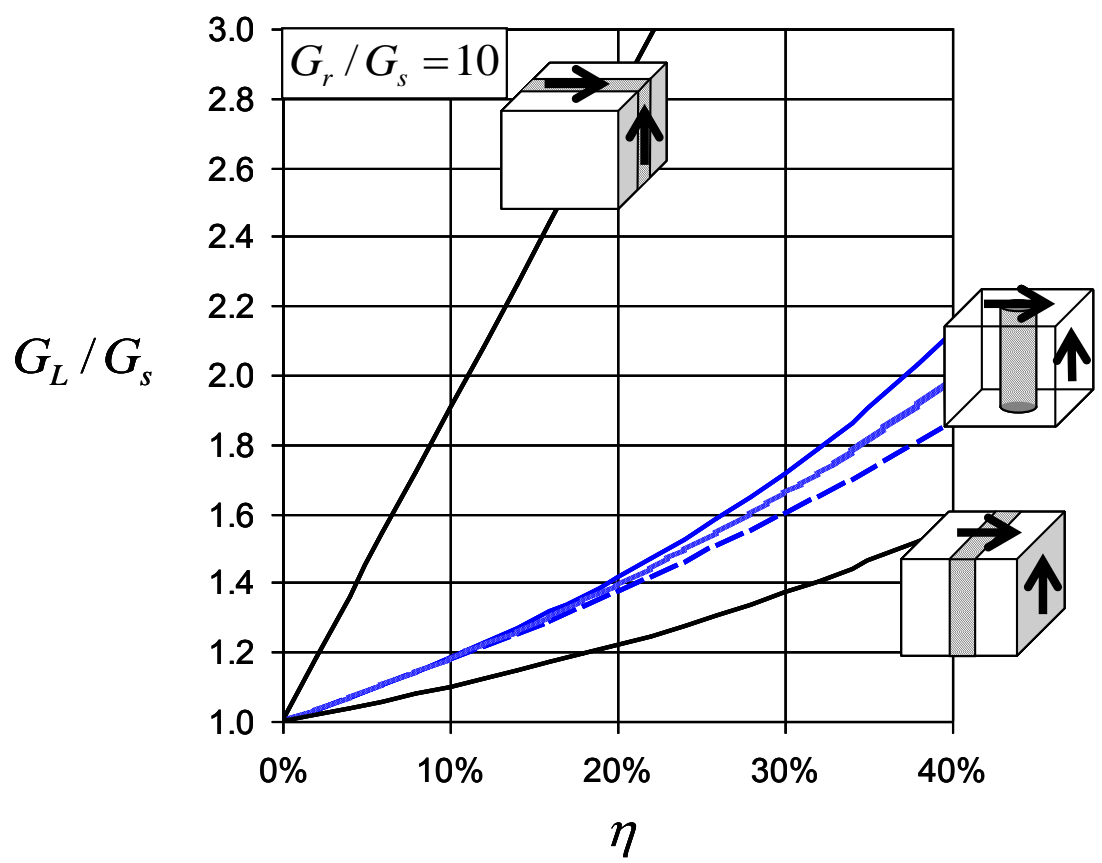

Figure 7. Increase of longitudinal shear stiffness for different types of reinforcement 


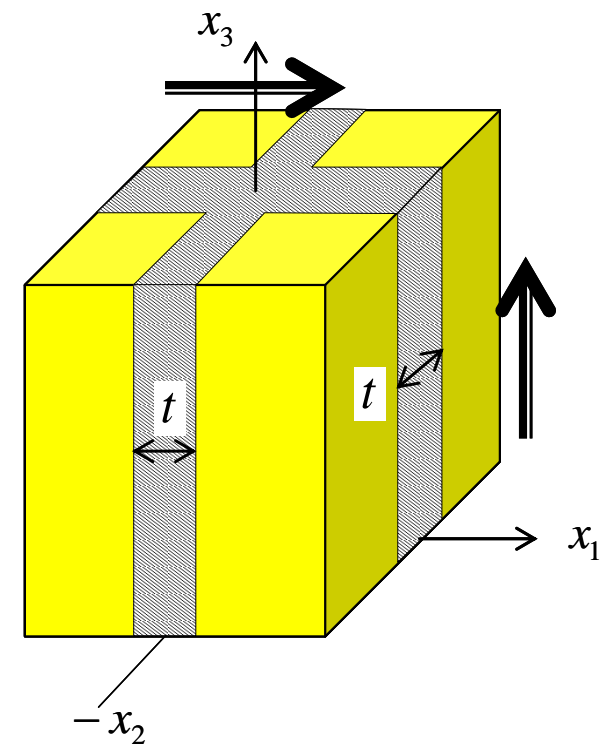

Figure 8. Unit cell for a cross trench configuration of reinforcement

Both variational methods already used for the column reinforcement technique, will now be implemented in order to provide sufficiently accurate bounds for the longitudinal shear modulus of cross trench reinforced soils.

\subsection{Upper bound estimate}

This upper bound estimate is obtained with the help of the following displacement field:

$$
\underline{\xi}^{\prime}=f\left(x_{1}\right) \underline{e}_{3}
$$

where function $f$ describing the displacement profile (Figure B1) is a piecewise linear continuous function defined as: 


$$
f\left(x_{1}\right)=-f\left(-x_{1}\right) \text { with } f\left(x_{1}\right)= \begin{cases}\left(\frac{\alpha}{t}\right) x_{1} & \text { for } 0 \leq x_{1} \leq t / 2 \\ \left(\frac{1-\alpha}{1-t}\right)\left(x_{1}-t / 2\right)+\alpha / 2 & \text { for } t / 2 \leq x_{1} \leq 1 / 2\end{cases}
$$

The implementation of the minimum principle of potential energy (16) making use of the above displacement field (see Appendix B for details) yields, after optimizing (i.e. minimizing) the bound with respect to parameter $\alpha$, the following upper bound:

$$
G_{L} \leq G_{L, \mathrm{ct}}^{\mathrm{ub}}=G_{r}\left[\frac{\sqrt{1-\eta}+\frac{G_{r}}{G_{s}}(1-\sqrt{1-\eta})}{\left((\eta-1+\sqrt{1-\eta})+\frac{G_{r}}{G_{s}}(2-\eta-\sqrt{1-\eta})\right)}\right]
$$

\subsection{Lower bound estimate}

The lower bound estimate is derived from the application of the complementary energy minimum principle (24), using the following piecewise constant shear stress field over the unit cell (Figure B2):

$$
\underline{\underline{\sigma}}^{\prime}=\tau\left(x_{2}\right)\left(\underline{e}_{1} \otimes \underline{e}_{3}+\underline{e}_{3} \otimes \underline{e}_{1}\right)
$$

where

$$
\tau\left(x_{2}\right)= \begin{cases}\tau_{1} & \text { for }-t / 2 \leq x_{2} \leq t / 2 \\ \tau_{2} & \text { for }-1 / 2 \leq x_{2} \leq-t / 2 \text { and } t / 2 \leq x_{2} \leq 1 / 2\end{cases}
$$

The introduction of this stress field into (24) provides, after maximization with respect to the two parameters $\tau_{1}$ and $\tau_{2}$, the following lower bound estimate for the longitudinal shear modulus of a cross trench reinforced soil (calculations are reported in Appendix B): 


$$
G_{L} \geq G_{L, \mathrm{ct}}^{\mathrm{lb}}=G_{s}\left[\frac{(1-\eta)}{(1-\eta)+(\sqrt{1-\eta}-(1-\eta)) G_{s} / G_{r}}+(1-\sqrt{1-\eta}) \frac{G_{r}}{G_{s}}\right]
$$

\subsection{Comparison with numerical estimates: column vs. cross trench configuration}

Figure 9 pictures a perspective view of the deformed configuration of half of the unit cell of a cross trench reinforced soil subject to longitudinal shear loading, resulting from a finite element simulation of the problem. As can be observed in this figure, the trench which is perpendicular to the loading plane undergoes much less deformations than the soil and the trench placed in the loading plane. This illustrates the fact that, contrary to the former, the latter trench fully contributes to the reinforced soil shear stiffness by means of the already mentioned "bracing effect".

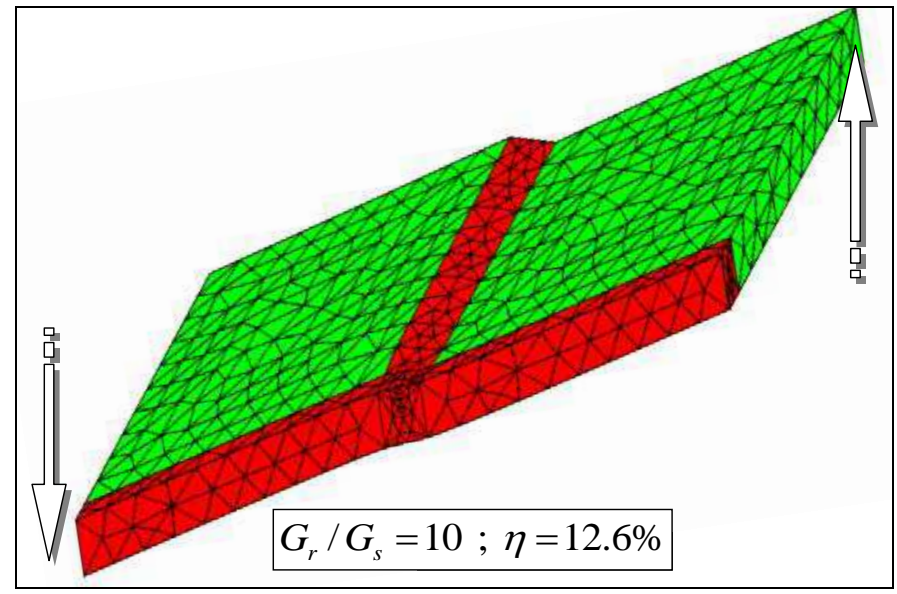

Figure 9. Deformed configuration of a cross trench reinforced soil under longitudinal shear loading

Figure 10 displays the analytical (bounds) and numerical results obtained for a cross trench reinforced soil. It shows that, as expected, the numerical estimates are comprised between the analytical bounds which are relatively close to each other, the mean value of 
these bounds (represented by the intermediate solid curve) providing an excellent estimate for the reinforced soil longitudinal shear stiffness.

Finally, all the results obtained in this section as well as in the two previous ones, have been summarized in Figure 11. By far the most remarkable result of the whole analysis, is the fact that reinforcing a soil according to the "cross trench technique" notably improves its performance in terms of overall longitudinal shear stiffness, in any case much more than a reinforcement by columnar inclusions. Indeed, for a typical value of $\eta=20 \%$, the cross trench configuration provides an increase of almost $110 \%$, that not so far from the maximum possible value of $180 \%$ (Voigt bound $(10=)$, to be compared with $40 \%$ for a reinforcement by column, the minimum value of $22 \%$ corresponding to the Reuss bound (15)).

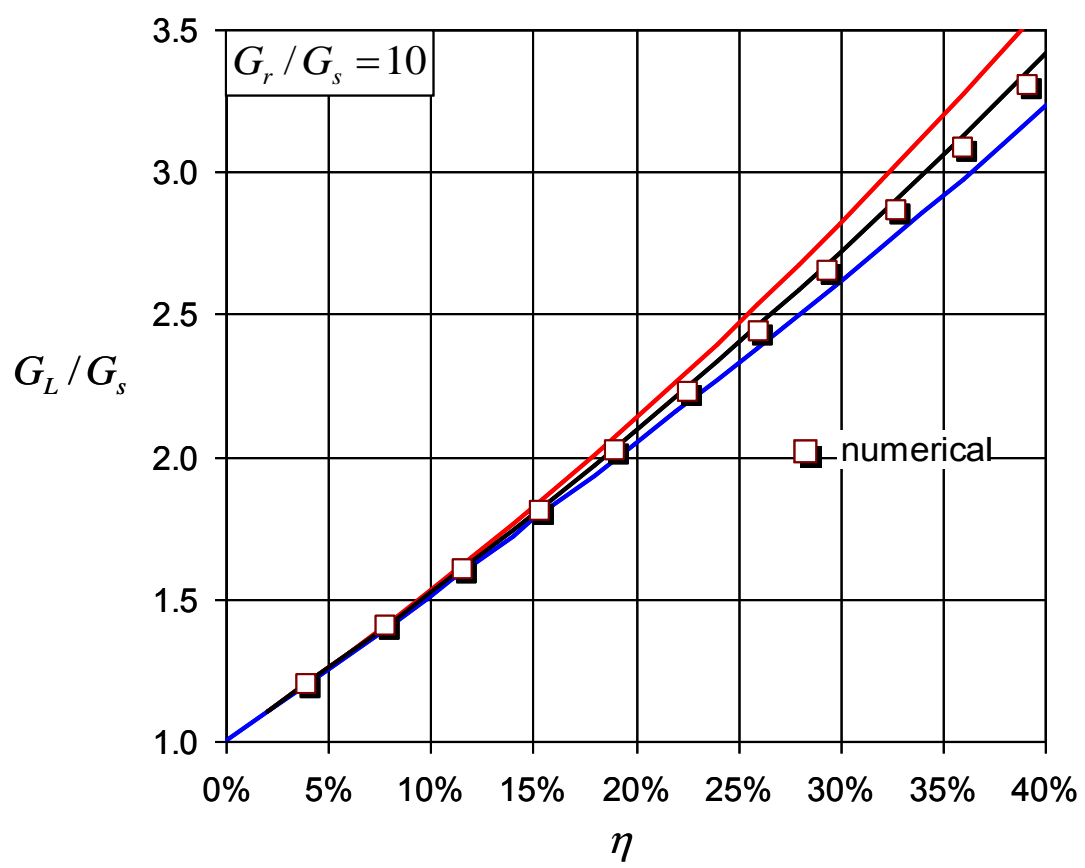

Figure 10. Analytical (bounds) and numerical estimates for the longitudinal shear stiffness of a cross trench reinforced soil 


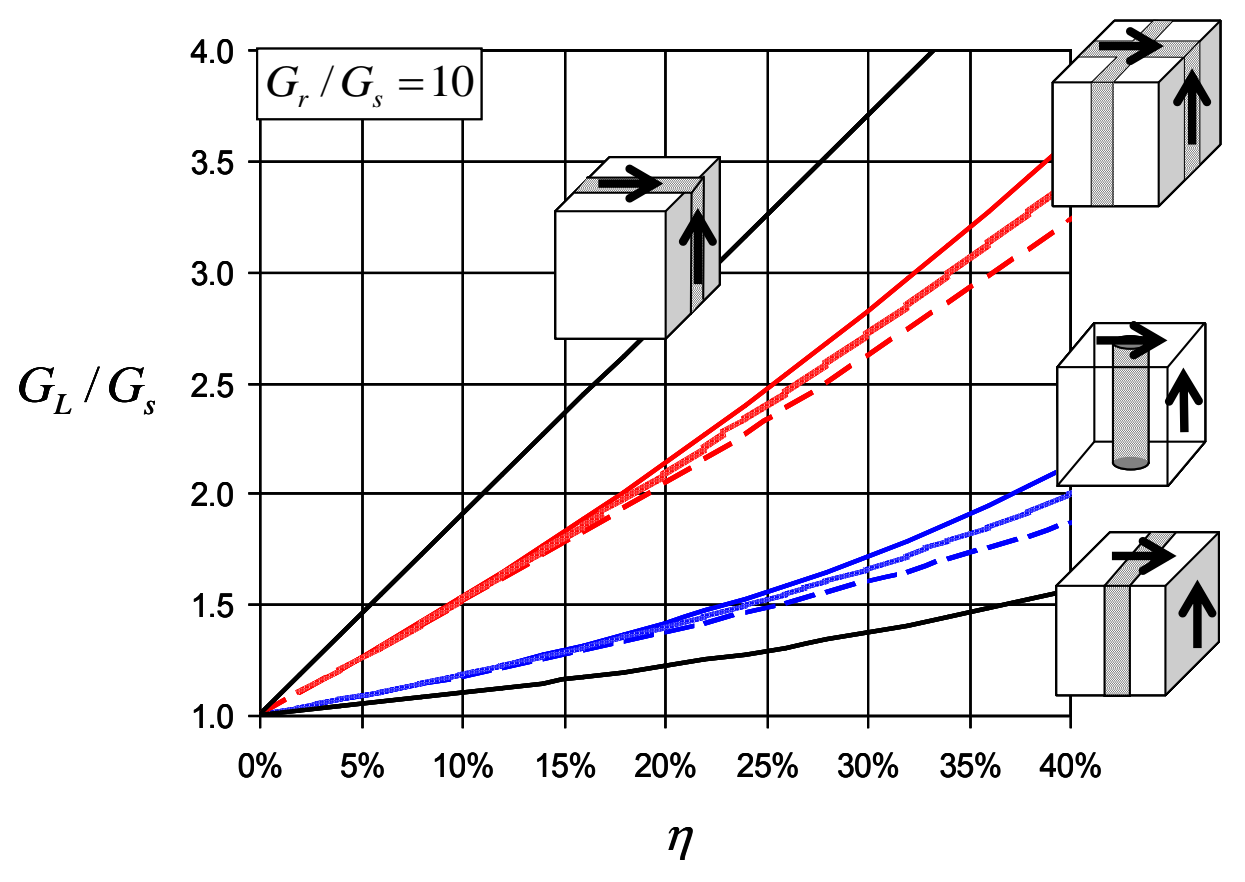

Figure 11. Longitudinal shear modulus of a reinforced soil for different configurations

\section{Application to the optimization of reinforcement layout as a way of reducing soil liquefaction}

\subsection{Problem statement}

As it is well known in geotechnical engineering, saturated relatively loose soils (sands or silts) subject to cyclic shear strains induced by earthquake ground motions, experience excess pore water pressure generation which results in a liquefaction phenomenon that is a collapse of their resistance. Our objective here is to examine, through a very simple model based upon the previously obtained homogenization results, to what extent such a phenomenon can be avoided from strengthening the soil by inclusions (example of stone or vibro-concrete columns: Baez and Martin, 1993; Adalier et al., 2003; Al-Homoud and Degen, 2006). 


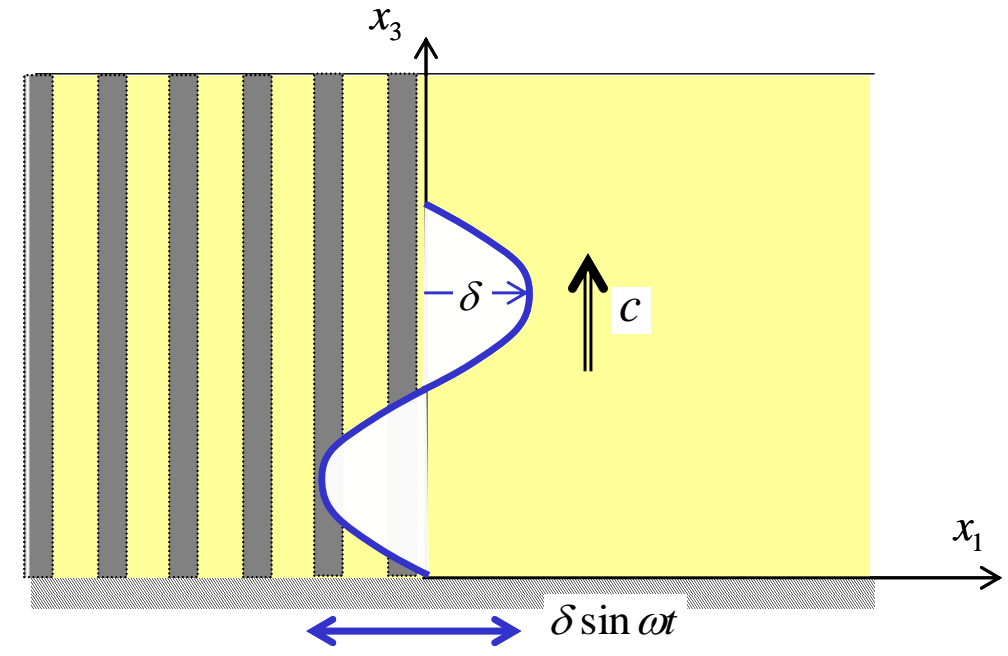

Figure 12. Vertical propagation of a seismic wave in a soil

The earthquake loading is schematized by a harmonic shear wave propagating upwards from the underlying substratum into the soil (Figure 12), defined as:

$$
\underline{\xi}(\underline{x}, t)=\delta \sin \omega\left(t-\frac{x_{3}}{c}\right) \underline{e}_{1}
$$

where $\delta$ is the horizontal displacement amplitude and $\omega$ the angular frequency, both being prescribed characteristics of the seismic loading, while $c$ is the shear wave velocity equal to:

$$
c_{s}=\sqrt{\frac{G_{s}}{\rho_{s}}} ; \rho_{\mathrm{s}}: \text { soil mass density }
$$

for the non reinforced soil and:

$$
c^{\text {hom }}=\sqrt{\frac{G_{L}}{\langle\rho\rangle}}
$$

for the reinforced soil, regarded as a homogenized elastic continuum, where $\langle\rho\rangle$ represents its average mass density defined as: 


$$
\langle\rho\rangle=(1-\eta) \rho_{s}+\eta \rho_{r}
$$

The cyclic strain field associated with (34) is therefore:

$$
\underline{\underline{\varepsilon}}(\underline{x}, t)=-\delta \frac{\omega}{2 c} \cos \omega\left(t-\frac{x_{3}}{c}\right)\left(\underline{e}_{1} \otimes \underline{e}_{3}+\underline{e}_{3} \otimes \underline{e}_{1}\right)
$$

so that the shear strain amplitude at any point is:

$$
\gamma=\delta \frac{\omega}{2 c}= \begin{cases}\gamma_{s}=\frac{\delta \omega}{2} \sqrt{\frac{\rho_{s}}{G_{s}}} & (\text { virgin soil) } \\ \gamma^{\text {hom }}=\frac{\delta \omega}{2} \sqrt{\frac{\langle\rho\rangle}{G_{L}}} & (\text { reinforced soil) }\end{cases}
$$

It thus appears that the ratio between the macroscopic shear strain amplitude of the reinforced soil and that of the original soil is:

$$
\frac{\gamma^{\text {hom }}}{\gamma_{s}}=\sqrt{\frac{G_{s}}{G_{L}}}
$$

where, for the sake of simplicity, it has been assumed that $\langle\rho\rangle \cong \rho_{s}$.

Actually, the relevant comparison to be performed is that between $\gamma_{s}$ and the average value of the shear strain in the soil of the unit cell subject to $\gamma^{\text {hom }}$, defined as:

$$
\underline{\underline{\epsilon}}=\gamma^{\mathrm{hom}}\left(\underline{e}_{1} \otimes \underline{e}_{3}+\underline{e}_{3} \otimes \underline{e}_{1}\right) \rightarrow\langle\gamma\rangle_{s}=\frac{1}{\left|C_{s}\right|} \int_{C_{s}} \varepsilon_{13} \mathrm{~d} V=\lambda \gamma^{\mathrm{hom}}
$$

where $C_{s}$ denotes the geometrical sub domain of the unit cell $C$ occupied by the soil, and $\lambda$ is a localization factor. 


\subsection{Assessing the mitigation of liquefaction risk}

The reduction of the potential risk of soil liquefaction to be expected from the stiffening effect of the reinforcement by inclusions, will be all the more important as the following ratio, which will conventionally be called risk reduction factor $R$, defined as:

$$
R=\frac{\langle\gamma\rangle_{s}}{\gamma_{s}}=\lambda \sqrt{\frac{G_{s}}{G_{L}}}
$$

will be smaller than unity. This factor is now evaluated for the different geometries of reinforcement examined above.

\subsubsection{Single trench reinforcement (multilayer model)}

Two cases should be considered depending on the orientation of the loading with respect to the direction of the reinforcing layer.

a) The plane of loading is parallel to the reinforcing trench (Figures $2(a)$ and $3(a)$ ). The shear strain field being homogeneous, equal to the macroscopic shear strain, the localization factor is equal to $\lambda^{=}=1$, and then from (10) and (42):

$$
R^{=}=\lambda^{=} \sqrt{\frac{G_{s}}{G_{L}^{=}}}=\left((1-\eta)+\eta \frac{G_{r}}{G_{s}}\right)^{-1 / 2}
$$

$b$ ) The plane of loading is normal to the reinforcing trench (Figures $2(b)$ and $3(b)$ ). The shear strain field being piecewise homogeneous (Eq. (12)), the localization factor is calculated from (12), (14) and (15) as:

$$
\lambda^{\perp}=\frac{\left(T / 2 G_{s}\right)}{\left[(1-\eta)\left(T / 2 G_{s}\right)+\eta\left(T / 2 G_{r}\right)\right]}=\frac{G_{L}^{\perp}}{G_{s}}
$$

hence 


$$
R^{\perp}=\lambda^{\perp} \sqrt{\frac{G_{s}}{G_{L}^{\perp}}}=\left((1-\eta)+\eta \frac{G_{s}}{G_{r}}\right)^{-1 / 2}
$$

\subsubsection{Reinforcement by circular columns}

Two different estimates of the localization factor can be obtained from the displacement and stress field used in Appendix A for the derivation of bounds on $G_{L}$. The "approximate" displacement field defined by (19) and (20) yields, after some calculations, the following estimate:

$$
\lambda_{\mathrm{col}}^{+}=\frac{1}{1-\eta}\left(1-\frac{2 \eta}{\left(1+G_{r} / G_{s}\right)+(4 \eta / \pi)\left(1-G_{r} / G_{s}\right)}\right)
$$

so that:

$$
R_{\mathrm{col}}^{+}=\lambda^{+} \sqrt{\frac{G_{s}}{G_{L, \mathrm{col}}^{\mathrm{ub}}}}
$$

On the other hand, the stress field defined by (A13)-(A15) makes it possible to calculate the following estimate for the localization factor:

$$
\lambda_{\mathrm{col}}^{-}=\frac{G_{L, \mathrm{col}}^{\mathrm{lb}}}{G_{s}}\left(\frac{1}{1-\eta}-\frac{\eta}{1-\eta} \frac{2 G_{r} / G_{s}}{\left(1+G_{r} / G_{s}\right)-(4 \eta / \pi)\left(1-G_{r} / G_{s}\right)}\right)
$$

and thus for the risk reduction factor:

$$
R_{\mathrm{col}}^{-}=\lambda_{\mathrm{col}}^{-} \sqrt{\frac{G_{s}}{G_{L, \mathrm{col}}^{\mathrm{b}}}}
$$

The variations of the two above estimates of the risk reduction factor as functions of the reinforcement volume fraction have been displayed in Figure 13, along with the evaluation of this factor deduced from the finite element simulations carried out in section 4. It can be seen from this figure that, even though the previous estimates cannot 
be rigorously interpreted as bounds on $R$, they actually bracket the numerical values (square symbols). Moreover, their average value (middle curve) provides a very accurate estimate for the reduction factor.

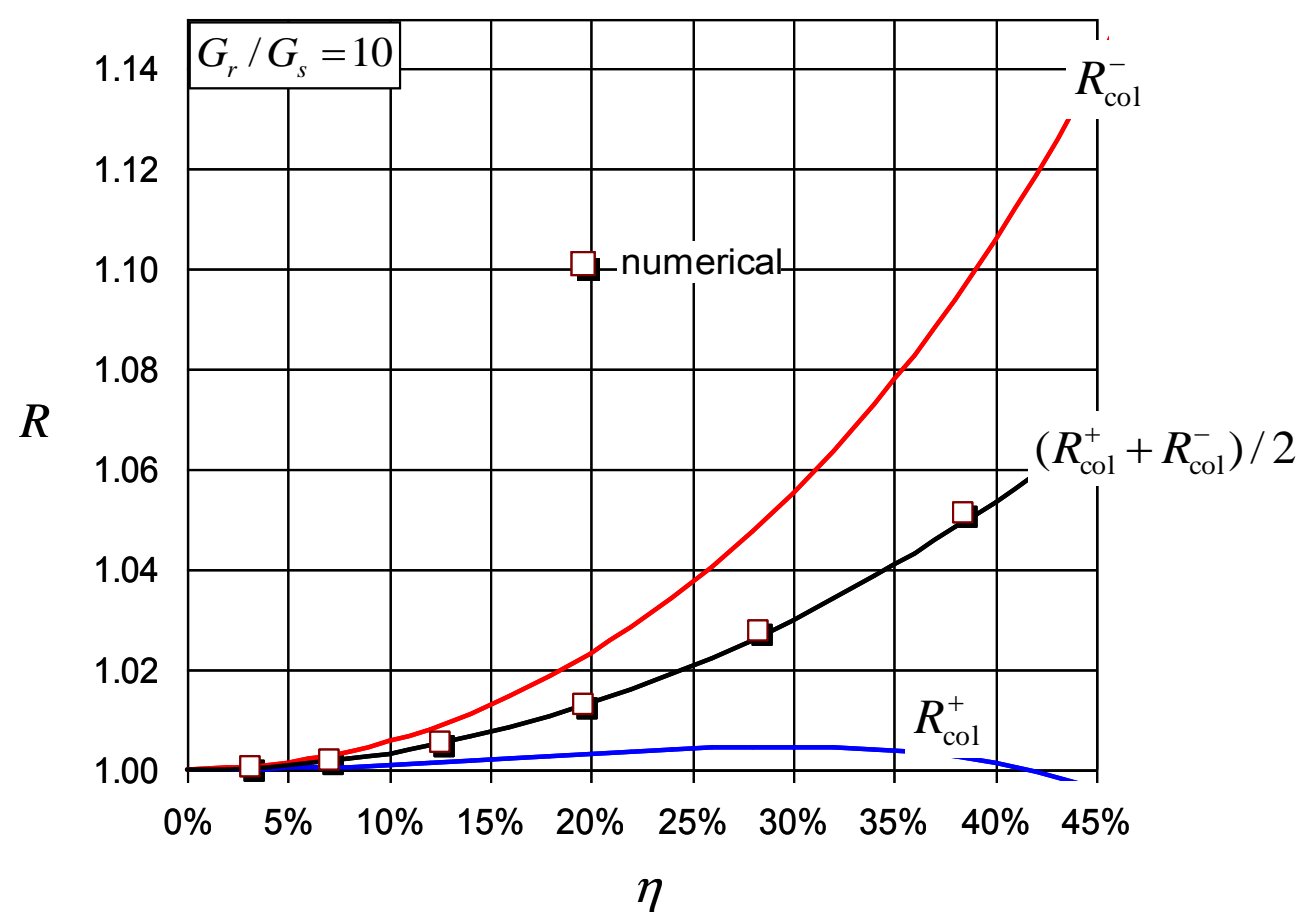

Figure 13. Evaluations of the liquefaction risk reduction factor for a column-reinforced soil

\subsubsection{Cross trench reinforcement}

The procedure is quite similar to that followed for a column-reinforced soil. Leaving aside detailed calculations, the optimized displacement field (29) used in the determination of the upper bound solution yields:

$$
\lambda_{\mathrm{ct}}^{+}=\left(2-\eta-\sqrt{1-\eta}+(\eta-1+\sqrt{1-\eta}) \frac{G_{s}}{G_{r}}\right)^{-1}
$$

and

$$
R_{\mathrm{ct}}^{+}=\lambda_{\mathrm{ct}}^{+} \sqrt{\frac{G_{s}}{G_{L, \mathrm{ct}}^{\mathrm{ub}}}}
$$


where the expression of $G_{L, \mathrm{ct}}^{\mathrm{ub}}$ is given by (30). Likewise, the optimized stress field (31)(32) used in the lower bound approach leads to: the following estimates of both the localization and risk reduction factors:

$$
\lambda_{\mathrm{ct}}^{-}=\left(\sqrt{1-\eta}+(1-\sqrt{1-\eta}) \frac{G_{s}}{G_{r}}\right)^{-1}, \quad R_{\mathrm{ct}}^{-}=\lambda_{\mathrm{ct}}^{-} \sqrt{\frac{G_{s}}{G_{L, \mathrm{ct}}^{\mathrm{lb}}}}
$$

with $G_{L, \mathrm{ct}}^{\mathrm{lb}}$ given by (33).

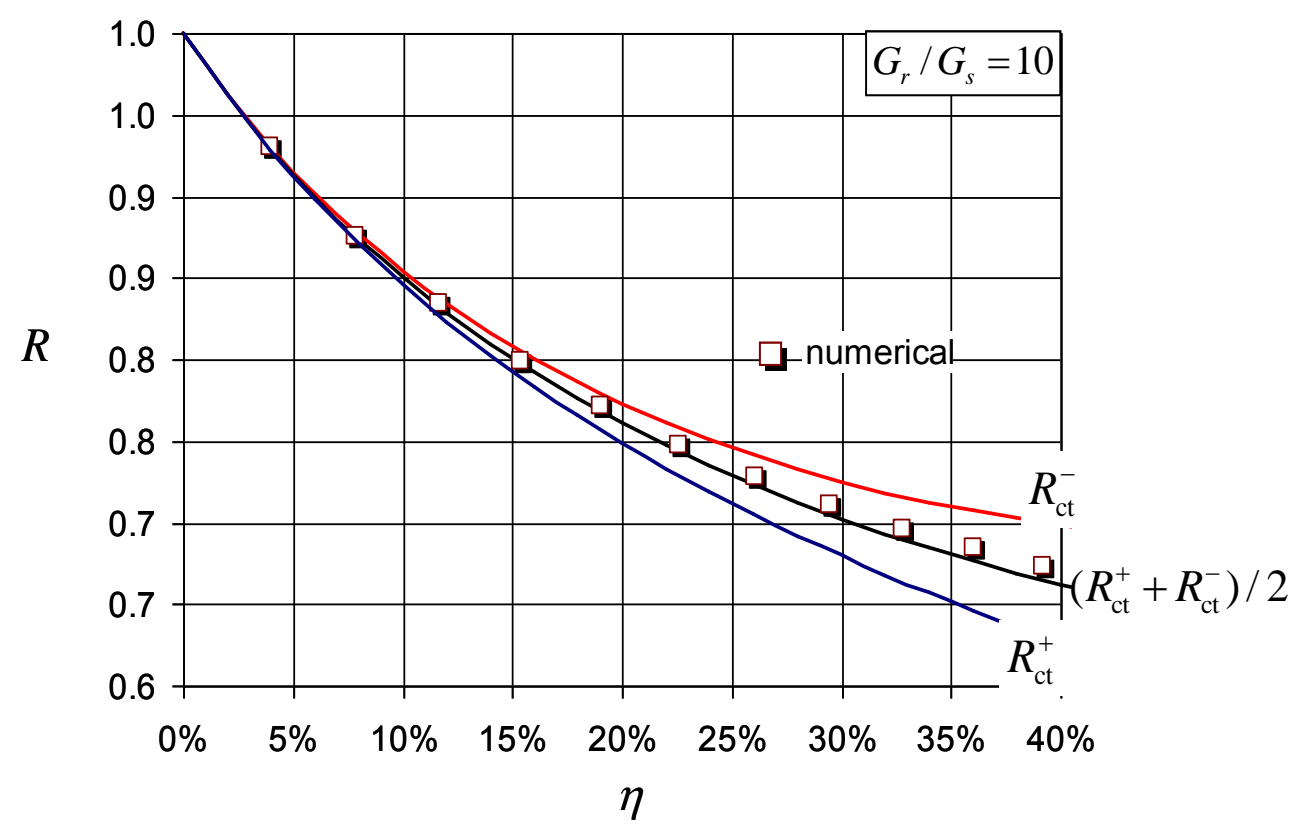

Figure 14. Evaluations of the liquefaction risk reduction factor for a cross trench reinforced soil

The above analytical estimates of the risk reduction factor are compared with the numerical ones in Figure 14. Again, it can be seen that the average value of these estimates provide an excellent evaluation of this factor. 


\section{Analysis of results and concluding remarks}

All the results obtained in the previous section are summarized in Figure 15 showing the variation of the risk reduction factor as a function of the reinforcement volume fraction for the different types of reinforcement: single trench, column or cross trench configurations. As it is clearly apparent from this figure, the cross sectional shape of the reinforcement has a considerable influence on its performance in terms of mitigation of liquefaction risk, due to the decrease of the cyclic shear strain amplitude experienced by the soil subject to seismic loading.

At first sight, referring to the bottom curve of Figure 15, one might wrongly conclude that reinforcing the soil by a single array of parallel trench would constitute the best way of reducing the risk of liquefaction. Unfortunately, the top curve of the same figure clearly shows this is only true when the trench orientation is in the seismic loading plane, the risk of liquefaction being on the contrary increased when the reinforcing trenches are normal to the same seismic loading plane.

The upper intermediate curves, which correspond to the most frequently used configuration of reinforcement by columnar inclusions, show that there is no such mitigation effect of liquefaction risk, a slight increase of this risk being even observed. This somewhat unexpected result can be explained as follows from the multiplicative decomposition (42) of factor $R$. Indeed, while the second square root term of this expression is obviously smaller than one, since the longitudinal shear stiffness is greater than the soil shear modulus (Figure 11), its potentially positive effect is completely wiped out by the fact that the localization factor $\lambda$ is larger than one, which means that the shear strains tend to concentrate in the soil due to the presence of the reinforcing inclusion. 


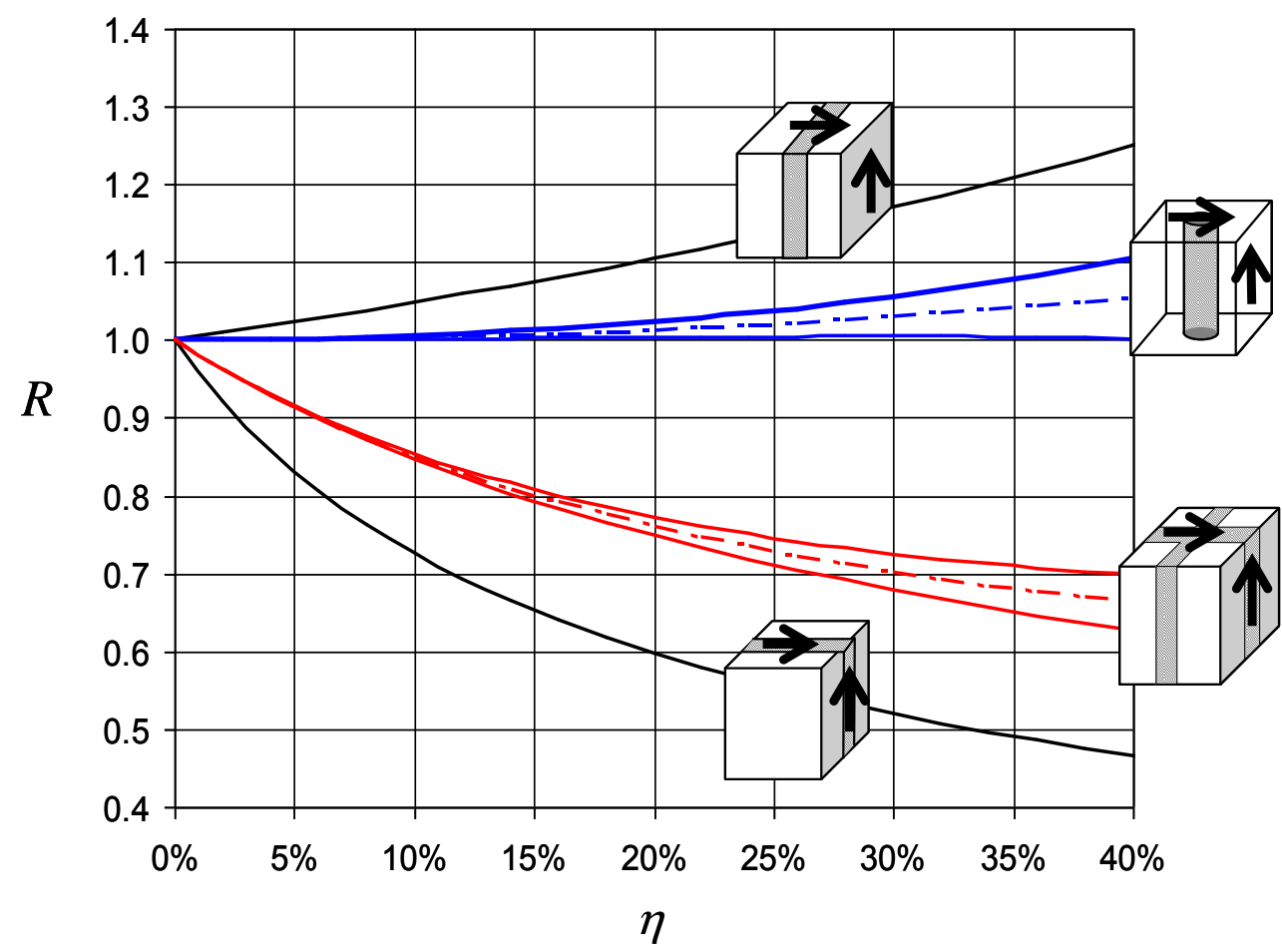

Figure 15. Evaluations of the liquefaction risk reduction factor for different kinds of reinforcements

It finally turns out that the cross trench configuration (lower intermediate curves) makes it possible to obtain a quite significant reduction of risk liquefaction, the more pronounced reinforcing effect being in such a case only partially obliterated by the strain concentration effect. This quite important result is corroborated by Figure 16 displaying the variations of the risk mitigation factor with respect to the reinforcement-soil relative shear stiffness, the reinforcement volume fraction being held at a constant typical value of $16 \%$. One could expect for instance that the risk factor would be cut in half when employing a sufficiently stiff reinforcing material.

The implementation of the homogenization theory for elastic periodic media has made it possible to derive rigorous and accurate estimates for the longitudinal shear stiffness of reinforced soils, as well as to determine the most effective configuration to be assigned to the cross section of the reinforcing inclusion. Among the key results of such 
an analysis, it should be pointed out that the cross trench configuration appears to be an optimized form both in terms of soil reinforcement efficiency and liquefaction risk remediation. This result has important consequences from an engineering design viewpoint, since it provides a strong theoretical background to the development of the cross trench reinforcement technique.

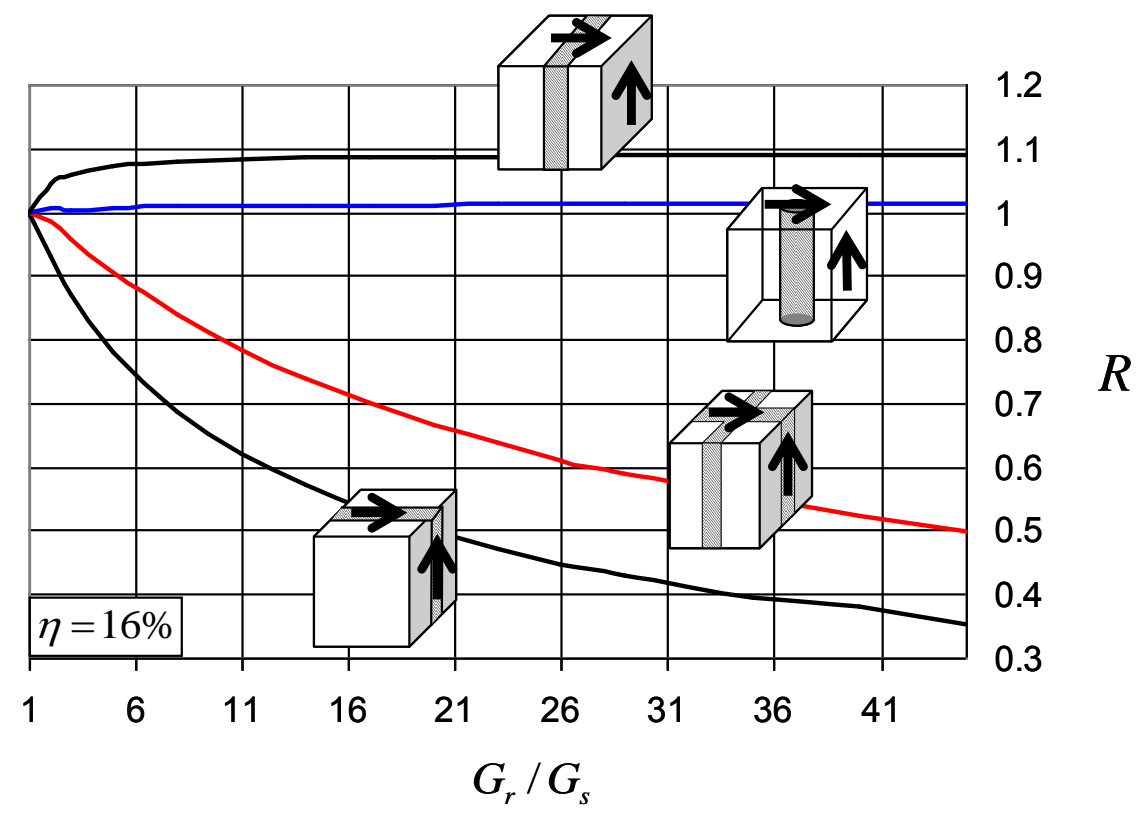

Figure 16. Liquefaction risk reduction factor as a function of the reinforcement relative shear stiffness for $\eta=16 \%$

\section{References}

Abdelkrim, M. and de Buhan, P. (2007). An elastoplastic homogenization procedure for predicting the settlement of a foundation reinforced by columns. European Journal of Mechanics, A/Solids, 26, pp. 736-757. 
Adalier, K., Elgamal, A., Meneses, J., Baez, J.I. (2003). Stone columns as liquefaction coutermeasure in non plastic silty soils. Soil Dyn. Earthquake Eng., 23, pp. 571-584.

Al-Homoud, A.S. and Degen, W. S. (2006). Marine stone columns to prevent earthquake induced soil liquefaction. Geotech. Geol. Eng., 24, pp. 775-790.

Baez, J.I. and Martin, G. (1993). Advances in the design of vibro systems for the improvement of liquefaction resistance. Proc. Symp. Ground Improvement, Vancouver Geotecnical Society, Canada, pp. 1-16.

Balaam, N.P. and Booker, J.R. (1981). Analysis of rigid rafts supported by granular piles. Int. J. Num Anal Meth Geomech., Vol 5, pp 379-403.

Bensoussan A., Lions J.L., Papanicolaou G. (1978) Asymptotic analysis for periodic structures. North-Holland Publishing Company: Amsterdam.

Canetta, G. and Nova, R. (1989). A numerical method for the analysis of ground improved by columnar inclusions. Computers and Geotechnics, Vol 7, pp 99-114.

Cast3M (2003). http://www.cast3m.cea.fr.

Duvaut G. (1976). Analyse fonctionnelle en mécanique des milieux continus. In Th. and Appl. Mech., ed. W. Koiter, North-Holland Publishing Company: Amsterdam, pp. 119-131.

Hashin Z. (1983). Analysis of composite materials-A survey. J. Appl. Mech., vol. 50, pp. 481-505.

Hashin, Z. and Rosen, B.W. (1964). The elastic moduli of fiber-reinforced materials. ASME, J. Appl. Mech., vol. 21, pp. 199-212. 
Hassen, G. and de Buhan, P. (2005). A two-phase model and related numerical tool for the design of soil structures reinforced by linear inclusions. European Journal of Mechanics A/ Solids, 24, pp. 987-1001

Hassen, G. Abdelkrim, M. and de Buhan, P. (2010). Finite element implementation of a homogenized constitutive law for stone-column reinforced foundation soils, with application to the design of structures. Computers and Geotechnics, 37, pp. 40-49

Jellali B., Bouassida, M. and de Buhan, P. (2005) A homogenization method for estimating the bearing capacity of soils reinforced by columns. Int. Num. Anal. Meth. Geomech, Vol 29, pp 1-16.

Jellali B., Bouassida, M. and de Buhan, P. (2011). Stability analysis of an embankment lying over a column reinforced soil. . Int. Num. Anal Meth Geomech., Vol. 35, pp. 12431256

Lee, J.S. and Pande, G.N. (1998) Analysis of stone-column reinforced foundations. Int. J. Num. Anal. Meth. Geomech. Vol 22, pp 1001-1020.

Sanchez-Palencia, E. (1980). Non homogeneous media and vibration theory. Lectures Notes in Physics, $\mathrm{n}^{\circ} 127$, Ed. Springer Verlag, Berlin.

Sudret, B. and de Buhan, P. (2001). Multiphase model for inclusion-reinforced geostructures. Applications to rock-bolted tunnels and piled raft foundations. Int. Num. Anal. Meth. Geomech., vol. 25, pp. 155-182.

Suquet, P. (1985). Elements of homogenization for inelastic solid mechanics. In: CISM Lecture Notes, $\mathrm{n}^{\circ} 272$, "Homogenization Techniques for Composite Media", SpringerVerlag: New York, pp. 193-278. 
$* * * * * * * * * *$ 


\section{Appendix A. Derivation of bounds on $G_{L}$ for a column-reinforced soil}

\section{$\diamond$ Upper bound estimate}

It should first be noted that the displacement field defined by (19) and (20) can be written in the form (2) that is:

$$
\underline{\xi}^{\prime}=\overbrace{\left(\underline{e}_{3} \otimes \underline{e}_{1}\right)}^{\underline{F}} \cdot \underline{x}+\overbrace{(\varphi(r)-r) \cos \theta \underline{e}_{3}}^{\underline{u}}
$$

since $\underline{u}(\underline{x})$ is equal to zero on the external boundary of the unit cell cross section, so that the periodicity condition is automatically satisfied. This implies that the displacement field is kinematically admissible with the following macroscopic strain:

$$
\underline{\underline{\epsilon}}=\overbrace{1 / 2}^{\Gamma}\left(\underline{e}_{1} \otimes \underline{e}_{3}+\underline{e}_{3} \otimes \underline{e}_{1}\right)
$$

On the other hand, referring to (18), the average value of the strain energy on the unit cell can be calculated as follows:

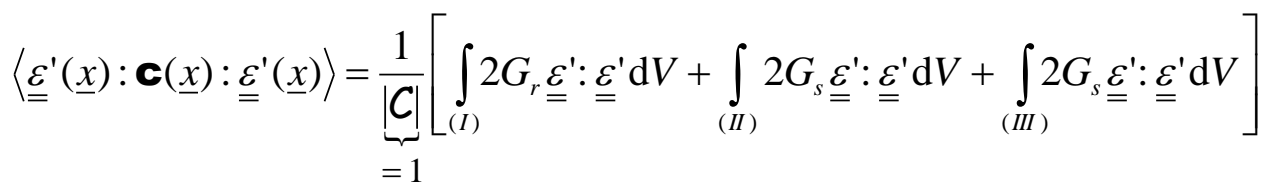

since $\operatorname{tr} \underline{\underline{\varepsilon}}=0$. Now, it turns out that the stress field $\underline{\underline{\sigma^{\prime}}}$ in zones $(I)$ and $(I I)$ associated with the displacement field $\underline{\xi}$ ' through the elastic constitutive law:

$$
\underline{\underline{\sigma^{\prime}}}=\left\{\begin{array}{c}
2 G_{r} \underline{\underline{\varepsilon^{\prime}}} \text { in zone }(I) \\
2 G_{s} \underline{\underline{\varepsilon^{\prime}}} \text { in zone }(I I)
\end{array}\right.
$$

satisfy the equilibrium conditions, including the continuity of the shear stress $\sigma_{r z}^{\prime}$ across the soil $(I I)$-column $(I)$ interface $(r=\rho)$. It follows from applying the virtual work principle (Clapeyron equation) that: 


$$
\int_{(I) \cup(I I)} \underset{\sigma^{\prime}:}{\underline{\varepsilon}^{\prime}} \mathrm{d} V=\int_{S(r=1 / 2)}\left(\underline{\underline{\sigma^{\prime}}} \cdot \underline{n}\right) \cdot \underline{\xi^{\prime}} \mathrm{d} S=\int_{S(r=1 / 2)} \sigma_{r z}^{\prime} \xi_{3}^{\prime} \mathrm{d} S
$$

where $S(r=1 / 2)$ is the interface between regions (II) and (III) (dashed circle in Figure 4) and from (19) and (20):

$$
\xi_{3}{ }^{\prime}(r=1 / 2)=1 / 2 \cos \theta ; \quad \sigma_{r z}^{\prime}(r=1 / 2)=G_{s} \varphi^{\prime}(1 / 2) \cos \theta
$$

We thus obtain:

$$
\int_{(I) \cup(I I)} \underset{\underline{\sigma^{\prime}}}{ }: \underline{\varepsilon}^{\prime} \mathrm{d} V=\frac{G_{s}}{4} \varphi^{\prime}(1 / 2) \int_{0}^{2 \pi}(\cos \theta)^{2} \mathrm{~d} \theta=\pi \frac{G_{s}}{4} \varphi^{\prime}(1 / 2)
$$

Meanwhile, the calculation of the elastic strain energy in zone (III) is straightforward, since in this region:

$$
\underline{\xi}^{\prime}=\overbrace{r \cos \theta}^{x_{1}} \underline{e}_{3} \Rightarrow \underline{\varepsilon}^{\prime}=1 / 2\left(\underline{e}_{1} \otimes \underline{e}_{3}+\underline{e}_{3} \otimes \underline{e}_{1}\right)
$$

and then

$$
\int_{(I I I)} 2 G_{s} \underline{\underline{\varepsilon^{\prime}}}: \underline{\varepsilon}^{\prime} \mathrm{d} V=G_{s}\left(1-\frac{\pi}{4}\right)
$$

The average of the elastic strain energy over the entire unit cell is therefore:

$$
\left\langle\underline{\underline{\varepsilon^{\prime}}}(\underline{x}): \mathbf{C}(\underline{x}): \underline{\underline{\varepsilon^{\prime}}}(\underline{x})\right\rangle=G_{s}\left[1+\frac{\pi}{4}\left(\varphi^{\prime}(1 / 2)-1\right)\right]
$$

with from (20):

$$
\varphi^{\prime}(1 / 2)=\frac{\left(G_{s}+G_{r}\right)+4 \rho^{2}\left(G_{r}-G_{s}\right)}{\left(G_{s}+G_{r}\right)-4 \rho^{2}\left(G_{r}-G_{s}\right)}, \quad \rho^{2}=\eta / \pi
$$

and thus from (18) and (A2):

$$
G_{L} \leq G_{s}\left[1+\frac{\pi}{4}\left(\frac{\left(G_{r}+G_{s}\right)+(4 \eta / \pi)\left(G_{r}-G_{s}\right)}{\left(G_{r}+G_{s}\right)-(4 \eta / \pi)\left(G_{r}-G_{s}\right)}-1\right)\right]
$$




\section{$\diamond$ Lower bound estimate}

The following stress field is defined in the same zones as the previous displacement field. It is associated in zones $(I)$ and (II) to the displacement field defined by (19) and (20), through the elastic law:

* Zone $(I)(0 \leq r \leq \rho)$

$$
\left\{\begin{array}{l}
\sigma_{r z}^{\prime}=2 G_{r} \varepsilon_{r z}^{\prime}=\frac{2 G_{s} G_{r}}{\left(G_{r}+G_{s}\right)-4 \rho^{2}\left(G_{r}-G_{s}\right)} \cos \theta \\
\sigma_{\theta z}^{\prime}=2 G_{r} \varepsilon_{\theta z}^{\prime}=-\frac{2 G_{s} G_{r}}{\left(G_{r}+G_{s}\right)-4 \rho^{2}\left(G_{r}-G_{s}\right)} \sin \theta
\end{array}\right.
$$

* Zone $(I I)(\rho \leq r \leq 1 / 2)$

$$
\left\{\begin{array}{l}
\sigma_{r z}^{\prime}=2 G_{s} \varepsilon_{r z}^{\prime}=G_{s} \frac{\left(G_{r}+G_{s}\right)+(\rho / r)^{2}\left(G_{r}-G_{s}\right)}{\left(G_{r}+G_{s}\right)-4 \rho^{2}\left(G_{r}-G_{s}\right)} \cos \theta \\
\sigma_{\theta z}^{\prime}=2 G_{s} \varepsilon_{\theta z}^{\prime}=-G_{s} \frac{\left(G_{r}+G_{s}\right)-(\rho / r)^{2}\left(G_{r}-G_{s}\right)}{\left(G_{r}+G_{s}\right)-4 \rho^{2}\left(G_{r}-G_{s}\right)} \sin \theta
\end{array}\right.
$$

while it is completed in zone (III) by:

$$
\left\{\begin{array}{l}
\sigma_{r z}^{\prime}=G_{s} \frac{\left(G_{r}+G_{s}\right)+4 \rho^{2}\left(G_{r}-G_{s}\right)}{\left(G_{r}+G_{s}\right)-4 \rho^{2}\left(G_{r}-G_{s}\right)} \cos \theta \\
\sigma_{\theta z}^{\prime}=-G_{s} \frac{\left(G_{r}+G_{s}\right)+4 \rho^{2}\left(G_{r}-G_{s}\right)}{\left(G_{r}+G_{s}\right)-4 \rho^{2}\left(G_{r}-G_{s}\right)} \sin \theta
\end{array}\right.
$$

It can be easily seen that the equilibrium equations are satisfied in each zone separately, while the shear stress component $\sigma_{r z}^{\prime}$ remains continuous across the interfaces between zones $(I)$ and (II) on the one hand, zones $(I I)$ and $(I I I)$ on the other hand. The macroscopic stress equilibrated by this stress field is: 


$$
\underline{\underline{\Sigma}}=\left\langle\underline{\underline{\sigma^{\prime}}}\right\rangle=\frac{1}{|C|} \int_{\partial C} \underline{x} \otimes \underline{\underline{\sigma}}^{\prime} \cdot \underline{n} \mathrm{~d} S=\overbrace{G_{s} \frac{\left(G_{r}+G_{s}\right)+4 \rho^{2}\left(G_{r}-G_{s}\right)}{\left(G_{r}+G_{s}\right)-4 \rho^{2}\left(G_{r}-G_{s}\right)}}^{T=G_{s} \varphi^{\prime}(1 / 2)} \otimes \underline{e}_{3}+\underline{e}_{3} \otimes \underline{e}_{1})
$$

The average elastic energy associated with the above statically admissible stress field $\underline{\underline{\sigma}}$ is:

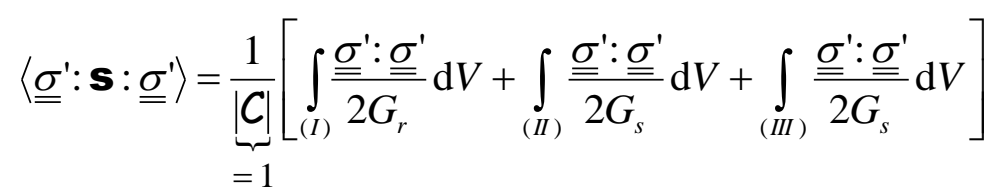

where the value of the sum of the first two terms is exactly the same as that calculated in (A7), that is on account of (A11):

$$
\int_{(I)}{\underline{\underline{\underline{\sigma^{\prime}}}}: \underline{\underline{\underline{\sigma}}}^{\prime}}_{2 G_{r}} \mathrm{~d} V+\int_{(I I)} \frac{{\underline{\underline{\sigma^{\prime}}}}^{\prime}{\underline{\underline{\sigma^{\prime}}}}^{\prime}}{2 G_{s}} \mathrm{~d} V=\int_{(I) \cup(I I)}{\underline{\underline{\sigma^{\prime}}}}^{\prime}: \underline{\underline{\varepsilon}}^{\prime} \mathrm{d} V=\pi \frac{G_{s}}{4} \varphi^{\prime}(1 / 2)=\frac{\pi}{4} T
$$

Since the stress field in zone (III) can be rewritten as:

$$
\underline{\underline{\sigma}}^{\prime}=T\left(\underline{e}_{1} \otimes \underline{e}_{3}+\underline{e}_{1} \otimes \underline{e}_{3}\right)
$$

the corresponding elastic stress energy is:

$$
\int_{(I I I)} \frac{{\underline{\underline{\sigma^{\prime}}}}^{\prime}:{\underline{\underline{\sigma^{\prime}}}}^{2}}{2 G_{s}} \mathrm{~d} V=\left(1-\frac{\pi}{4}\right) \frac{T^{2}}{G_{s}}
$$

so that:

$$
\left\langle\underline{\underline{\sigma^{\prime}}}: \mathbf{s}: \underline{\underline{\sigma^{\prime}}}\right\rangle=\left(1-\frac{\pi}{4}\right) \frac{T^{2}}{G_{s}}+\frac{\pi}{4} T
$$

As a result of (24), (A16) and (A21), the following lower bound is finally obtained:

$$
G_{L} \geq T^{2}\left[\left(1-\frac{\pi}{4}\right) \frac{T^{2}}{G_{s}}+\frac{\pi}{4} T\right]^{-1}=G_{s}\left[\left(1-\frac{\pi}{4}\right)+\frac{\pi}{4}\left(\varphi^{\prime}(1 / 2)\right)^{-1}\right]^{-1}
$$


that is:

$$
G_{L} \geq G_{s}\left[\left(1-\frac{\pi}{4}\right)+\frac{\pi}{4}\left(\frac{\left(G_{r}+G_{s}\right)-(4 \pi / \eta)\left(G_{r}-G_{s}\right)}{\left(G_{r}+G_{s}\right)+(4 \pi / \eta)\left(G_{r}-G_{s}\right)}\right)\right]^{-1}
$$

Remark. It should noted that the previously mentioned displacement and stress fields restricted to zones $(I)$ and $(I I)$ correspond to the elastic solution exhibited by Hashin and Rosen (1964) for their axisymmetric composite cell model (see 3.3.).

\section{Appendix B. Derivation of bounds on $G_{L}$ for a cross trench reinforced soil}

\section{$\diamond$ Upper bound estimate}

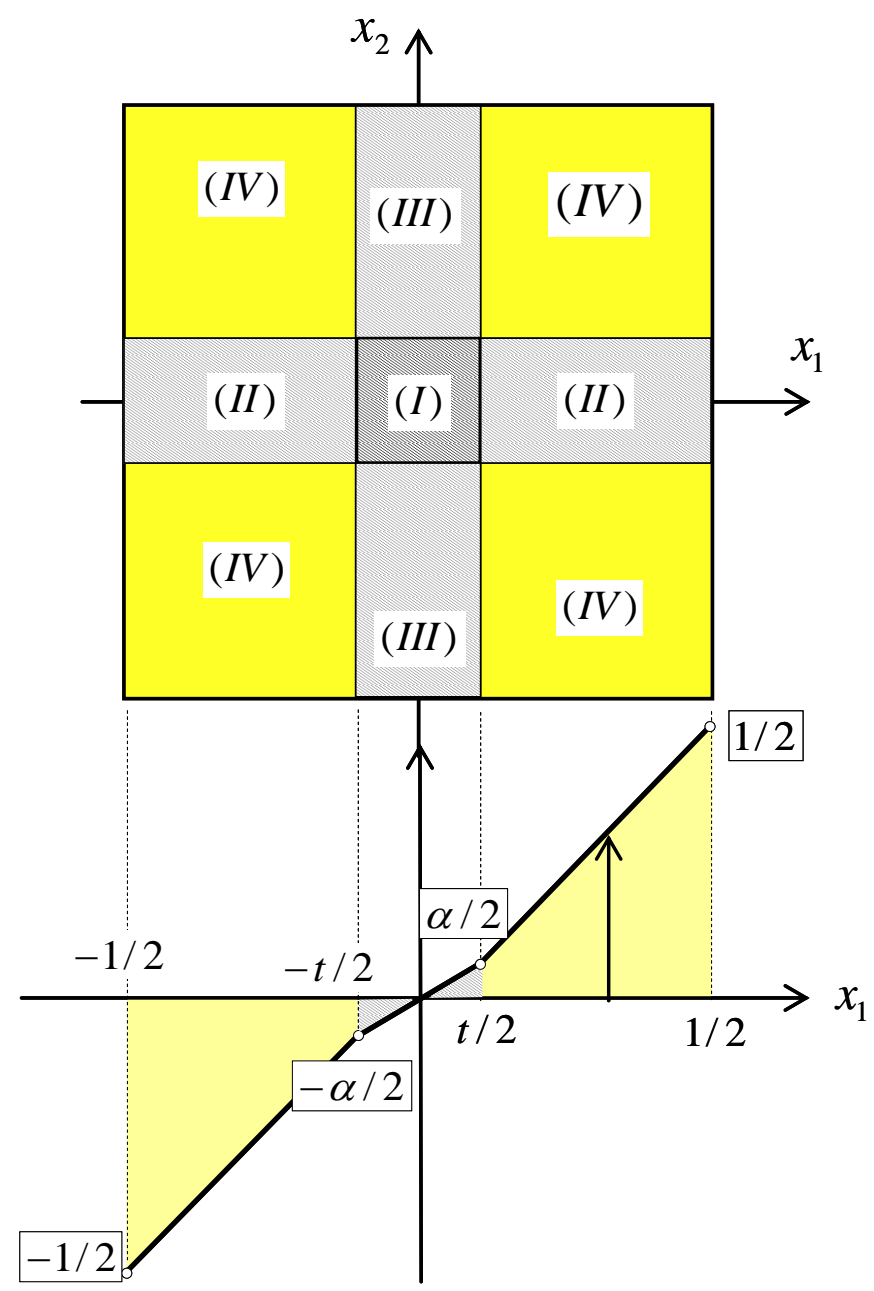

Figure B1. Displacement profile in the unit cell 
Since the displacement field defined by (28) and (29) can be rewritten as:

$$
\underline{\xi^{\prime}}=\overbrace{\left(\underline{e}_{3} \otimes \underline{e}_{1}\right)}^{\underline{F}} \cdot \underline{x}+\overbrace{\left(f\left(x_{1}\right)-x_{1}\right) \underline{e}_{3}}^{\underline{u}(x)}
$$

where $\underline{u}(\underline{x})$, being equal to zero on the external boundary of the unit cell cross section, is periodic, it appears to be kinematically admissible with the following macroscopic strain:

$$
\underline{\underline{\epsilon}}=\overbrace{1 / 2}^{\Gamma}\left(\underline{e}_{1} \otimes \underline{e}_{3}+\underline{e}_{3} \otimes \underline{e}_{1}\right)
$$

The associated strain field is therefore:

$$
\underline{\varepsilon}^{\prime}=\frac{f^{\prime}\left(x_{1}\right)}{2}\left(\underline{e}_{1} \otimes \underline{e}_{3}+\underline{e}_{3} \otimes \underline{e}_{1}\right) \text { with } f^{\prime}\left(x_{1}\right)= \begin{cases}\frac{\alpha}{t} & \text { for }\left|x_{1}\right| \leq t / 2 \\ \frac{1-\alpha}{1-t} & \text { for } t / 2 \leq\left|x_{1}\right| \leq 1 / 2\end{cases}
$$

so that:

$$
2 \underline{\underline{\varepsilon}}^{\prime}: \underline{\underline{\varepsilon^{\prime}}}= \begin{cases}\left(\frac{\alpha}{t}\right)^{2} & \text { for }\left|x_{1}\right| \leq t / 2 \\ \left(\frac{1-\alpha}{1-t}\right)^{2} & \text { for } t / 2 \leq\left|x_{1}\right| \leq 1 / 2\end{cases}
$$

and then, referring to the zoning of Figure (B1):

$$
\begin{aligned}
& \left\langle\underline{\underline{\varepsilon}}^{\prime}(\underline{x}): \mathbf{C}(\underline{x}): \underline{\underline{\varepsilon^{\prime}}}(\underline{x})\right\rangle=\frac{1}{|C|}\left[\int_{(I) \cup(I I I)} 2 G_{r} \underline{\underline{\varepsilon^{\prime}}}: \underline{\underline{\varepsilon}}^{\prime} \mathrm{d} V+\int_{(I I)} 2 G_{r} \underline{\underline{\varepsilon}}^{\prime}: \underline{\underline{\varepsilon}}^{\prime} \mathrm{d} V+\int_{(I V)} 2 G_{s} \underline{\underline{\varepsilon^{\prime}}}: \underline{\underline{\varepsilon}}^{\prime} \mathrm{d} V\right] \\
& =1 \\
& =G_{r}\left(\frac{\alpha}{t}\right)^{2} t+G_{r}\left(\frac{1-\alpha}{1-t}\right)^{2}\left(t-t^{2}\right)+G_{s}\left(\frac{1-\alpha}{1-t}\right)^{2}(1-t)^{2}
\end{aligned}
$$

The upper bound estimate deduced from the general inequality (18):

$$
G_{L} \leq G_{r} \frac{\alpha^{2}}{t}+G_{r}(1-\alpha)^{2} \frac{t}{1-t}+G_{s}(1-\alpha)^{2}=g(\alpha)
$$

is then minimized with respect to parameter $\alpha$ : 


$$
G_{L} \leq \operatorname{Min}_{\alpha} g(\alpha)=g\left(\alpha=\frac{t \bar{G}}{t \bar{G}+(1-t) G_{r}}\right)=G_{r} \frac{\bar{G}}{t \bar{G}+(1-t) G_{r}}
$$

where $\bar{G}=(1-t) G_{s}+t G_{r}$.

Taking into account the relationship between the trench thickness and the reinforcement volume fraction:

$$
\eta=t(2-t) \rightarrow t=1-\sqrt{1-\eta}
$$

the optimized bound (30) is finally obtained.

\section{$\diamond$ Lower bound estimate}

The stress field defined by (31) and (32) is statically admissible, since it obviously satisfies the equilibrium equation in each zone (Figure (B2)), where it is constant, while the stress vector continuity is ensured at the interfaces between these zones (namely between zones $(I) \cup(I I)$ and $(I I I) \cup(I V))$. Furthermore it equilibrates the following macroscopic stress:

$$
\underline{\underline{\Sigma}}=\left\langle\underline{\underline{\sigma}}^{\prime}\right\rangle=T\left(\underline{e}_{1} \otimes \underline{e}_{3}+\underline{e}_{3} \otimes \underline{e}_{1}\right) \text { with } T=(1-t) \tau_{2}+t \tau_{1}
$$

The calculation of the complementary energy yields

$$
\begin{aligned}
& \left\langle\underline{\underline{\sigma^{\prime}}: \mathbf{s}}: \underline{\underline{\sigma^{\prime}}}\right\rangle=\frac{1}{|C|}\left[\int_{(I) \cup(I I)} \frac{{\underline{\underline{\sigma^{\prime}}}}^{\prime}:{\underline{\underline{\sigma^{\prime}}}}^{2}}{2 G_{r}} \mathrm{~d} V+\int_{(I I I)} \frac{{\underline{\underline{\sigma^{\prime}}}}^{\prime}:{\underline{\underline{\sigma^{\prime}}}}^{2}}{2 G_{r}} \mathrm{~d} V+\int_{(I V)} \frac{{\underline{\underline{\sigma^{\prime}}}}^{\prime}:{\underline{\underline{\sigma^{\prime}}}}^{\prime}}{2 G_{s}} \mathrm{~d} V\right] \\
& =1 \\
& =t \frac{\tau_{1}^{2}}{G_{r}}+\left(t-t^{2}\right) \frac{\tau_{2}^{2}}{G_{r}}+(1-t)^{2} \frac{\tau_{2}^{2}}{G_{s}}=F\left(\tau_{1}, \tau_{2}\right)
\end{aligned}
$$




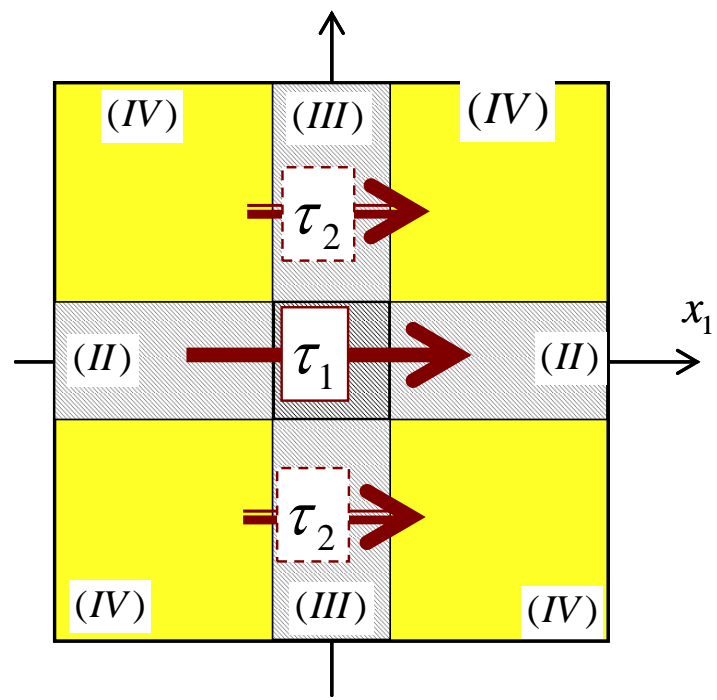

Figure B2. Piecewise homogeneous shear stress field in the unit cell

hence the following lower bound:

$$
G_{L} \geq T^{2} / F\left(\tau_{1}, \tau_{2}\right)
$$

Searching for the maximum of the right hand member of (B11) with respect to $\tau_{1}$ and $\tau_{2}$, under the condition $T=t \tau_{1}+(1-t) \tau_{2}$, yields:

$$
G_{L} \geq G_{s}\left[\frac{1-t}{(1-t)+t G_{s} / G_{r}}+t \frac{G_{r}}{G_{s}}\right]
$$

that is:

$$
G_{L} \geq G_{s}\left[\frac{(1-\eta)}{(1-\eta)+(\sqrt{1-\eta}-(1-\eta)) G_{s} / G_{r}}+(1-\sqrt{1-\eta}) \frac{G_{r}}{G_{s}}\right]
$$

